\title{
COMBINING THE INCOMPATIBLE AND STERILE INSECT TECHNIQUES FOR PEST AND VECTOR CONTROL
}

\author{
L. A. BATON ${ }^{1}$, D. ZHANG ${ }^{2}$, Y. LI $^{2}$ AND Z. XI ${ }^{1,2}$ \\ ${ }^{1}$ Department of Microbiology and Molecular Genetics, Michigan State University, \\ East Lansing, Michigan 48824,USA; xizy@msu.edu \\ ${ }^{2}$ Key Laboratory of Tropical Disease Control of the Ministry of Education, \\ Sun Yat-sen University - Michigan State University Joint Center of Vector Control \\ for Tropical Diseases, Zhongshan School of Medicine, Sun Yat-Sen University, \\ Guangzhou, Guangdong 510080, China
}

\begin{abstract}
SUMMARY
The Incompatible Insect Technique (IIT) is a Sterile Insect Technique (SIT)-related approach that uses the reproductive parasitism caused by infection with maternally-inherited bacterial endosymbionts to make released males reproductively incompatible with the wild-type females of the target population. The most common and widespread of such endosymbionts is Wolbachia, which is found throughout many insect orders, and often causes cytoplasmic incompatibility (CI), a form of conditional sterility where the fertilized eggs of females not infected with the same Wolbachia strain as the males with which they are mated undergo embryonic death. An advantage of IIT is that the incompatibility induced by Wolbachia often has either no or only minor effects on the quality of infected males. In addition, such endosymbionts can also have other desirable phenotypic effects on their hosts, such as reducing the ability of target species to act as disease vectors, thus allowing the undesirable sex(es) to be tolerated among the sterile insects to be released. However, an inherent problem with IIT, which has so far restricted its operational use, is that, unlike SIT, the accidental release of endosymbiont-infected females may prevent further population suppression by causing unintended population replacement, whereby the original target population is replaced with individuals infected with the same endosymbiont strain as the released males. A solution to this problem, at least for the majority of insects whose females are more sensitive than males to radiation, is to combine IIT with SIT, such that all endosymbiont-infected individuals destined for release are also first subjected to low-dose radiation, which completely sterilizes any contaminant females without affecting the incompatibility or quality of the irradiated males. Here, we discuss the biology and general theoretical principles underlying the use of IIT alone, and the rationale and necessity of combining IIT with SIT, as well as the logistical problems encountered, and technological developments required, for the massproduction and release of irradiated endosymbiont-infected individuals as part of area-wide integrated pest control programmes. We primarily illustrate our discussion with examples involving mosquitoes, for which the majority of the relevant research has been conducted, including the first open-release field trial of combined IIT/SIT application against the important arboviral vector Aedes albopictus (Skuse). However,
\end{abstract} Development and Field Application, pp. 367-404. CRC Press, Boca Raton, Florida, USA. 
the combined IIT/SIT approach should be broadly applicable to a wide range of other insect pests and vectors, and so of interest to entomologists in general.

Key Words: Aedes, Wolbachia, combined IIT/SIT, cytoplasmic incompatibility, symbiosis, arboviruses, mosquitoes, radiation, vector-borne diseases

\section{INTRODUCTION}

The irradiation-based Sterile Insect Technique (SIT) has been successfully used to suppress the populations of a number of insect pests and vectors of agricultural and veterinary importance (Dyck et al. 2021). However, the application of the SIT against other important groups of insects, especially the mosquito vectors of human pathogens, has, so far, been limited (Benedict and Robinson 2003; Dame et al. 2009; Bourtzis et al. 2016; Scott and Benedict 2016). There are various reasons for this, and they have been debated, but the development and implementation of the SIT for such insects continues, and is still an active and productive area of research, and it is hoped that, with further investigation and optimization, the SIT can be successfully and operationally deployed against mosquitoes in the not so distant future (Alphey et al. 2010; Bourtzis et al. 2016; Lees et al. 2015, 2021).

Concurrently, however, other approaches have also been, and continue to be, explored (Alphey 2014; Scott and Benedict 2016). One alternative approach is the Incompatible Insect Technique (IIT), which uses infection with maternally-inherited prokaryotic endosymbionts, such as the alpha-proteobacterium Wolbachia, to suppress host populations. Like other sterile-male-based methods of population suppression, these endosymbionts make the released males reproductively incompatible with wild-type females in the target population.

In this chapter, we outline the biological and theoretical basis of endosymbiontmediated IIT and argue why it is an attractive alternative to the SIT for some groups of insects. However, in the absence of a perfect sex separation system, IIT application has a fundamental constraint necessitating its combination with the SIT. Our discussion primarily concerns mosquitoes, but there is an increasingly large research literature on endosymbionts causing reproductive parasitism in a wide range of insect hosts, which readers are encouraged to explore for themselves.

\section{THE STERILE INSECT TECHNIQUE (SIT)}

Historically, there have been a number of laboratory tests and pilot field trials of irradiation-based SIT against mosquitoes (Benedict and Robinson 2003; Dame et al. 2009). Despite this, the SIT against mosquitoes has not yet been deployed operationally on a larger scale. Various reasons have been given for this, such as the failure to effectively optimize the timing and magnitude of the irradiation dose used on mass-reared individuals, the high rate of intrinsic increase of these insects and the failure to release sufficient males, an inability to efficiently and cost-effectively massproduce and/or release them, a lack of knowledge regarding the basic biology and ecology of the target species, and inadequate methods of sex separation (Dame et al. 2009; Scott and Benedict 2016). 
More recently, interest in using the SIT against mosquitoes has been revived, and the positive results of small-scale field trials (Bellini et al. 2007, 2013b) support the notion that the SIT is feasible against mosquitoes. In addition, over the past decade, the Food and Agriculture Organization of the United Nations (FAO) and the International Atomic Energy Agency (IAEA), in response to increasing requests from their member states, have increased their efforts to explore and disseminate the possibilities of integrating the SIT within AW-IPM approaches to manage better mosquito populations (Bourtzis et al. 2016; Lees et al. 2013, 2015; 2021).

\subsection{Reduced Quality of Irradiation-sterilized Insects}

A requirement of the SIT is that radiation-based sterilisation does not have serious adverse effects on male competitiveness or overall quality (Knipling 1955). Such effects may derive either from the direct deleterious effects of radiation itself, or indirectly through the mass-rearing and handling procedures and ambient conditions required for the administration of radiation (Bourtzis and Robinson 2006; Bakri et al. 2021). Insects vary in their radio-sensitivity, with some species being inherently more sensitive to the effects of irradiation, such that irradiation doses inducing high levels of male sterility often also have appreciable negative effects on male quality (e.g. mating competitiveness and survival) for some insect species (Bakri et al. 2005; Helinski et al. 2009). For mosquitoes, the process of irradiation has frequently been reported to reduce male competitiveness and survival (Arunachalam and Curtis 1985; Dame et al. 2009; Helinski et al. 2009; Oliva et al. 2012; Maïga et al. 2014; Yamada et al. 2014a, 2014b; Zhang et al. 2016; Zheng et al. 2019). However, whether such negative effects are due to the radiation itself, or the conditions and procedures under which the radiation is administered is often unclear (Scott and Benedict 2016; Yamada et al. 2019). The latter can impose significant fitness costs independent of the effects of radiation itself, which can reduce their quality for use in the SIT.

Many pest/vector species are fragile, with complex holometabolous life cycles, complicating their handling and irradiation, especially under the conditions of massrearing and mass-release required for the SIT. Different life cycle stages may also vary in their radio-sensitivity (e.g. late pupae versus adults), and careful timing of irradiation can help to minimize radiation-induced damage, as well as maximize sexspecific differences in radio-sensitivity, which is important for sterilizing contaminant females without adversely affecting male quality (see Section 4.2) (Andreasen and Curtis 2005; Helinski et al. 2006; Brelsfoard et al. 2009; Balestrino et al. 2010; Ndo et al. 2014; Zhang et al. 2015b).

Regardless of whether the adverse effects of irradiation are direct or indirect - and whether or not it might be possible in future to ameliorate such effects through optimization of irradiation protocols and development of better technology - for some groups of insects there is currently a necessary trade-off between sterility and quality, such that, as higher irradiation doses increase male sterility, they simultaneously decrease male quality (Helinski and Knols 2008; Balestrino et al. 2010; Bellini et al. 2013a). In many instances, intermediate irradiation doses can be identified that provide an optimal balance between male sterility and quality (Parker and Mehta 2007; Helinski et al. 2009). Consequently, the use of the SIT may not be precluded 
(Bellini et al. 2007, 2013b; Scott and Benedict 2016), although its overall efficiency may be reduced, and its cost-effectiveness decreased, through necessitating larger numbers of insects to be produced and released during area-wide control programmes. For target insects with very high reproductive potential, like some mosquito species (Alphey et al. 2010), the problem of trading-off residual fertility against male quality could be particularly acute, because population can rebound easily through those survived eggs as seeds. With low residual fertility, the number of emerging adults in the wild may be relatively high as the low number of hatching eggs is compensated by low competition for resources among surviving larvae, and hence higher survival rates during the development. Therefore, minimum levels of sterility are necessarily required to overcome the intrinsic growth of the target population (Barclay 2021).

\subsection{Imperfect Sex Separation}

Another problem for the implementation of the SIT, as well as all other sterile-insectbased methods, against insects like mosquitoes where adult females (and not males) of the target species are the pests/vectors, is the absence of perfect sex separation methods (Gilles et al. 2014). Thus, the release of females, even as relatively small numbers of contaminant individuals, is considered unacceptable for SIT applications to control those pests/vectors due to the risk of increased crop destruction, parasitism or pathogen transmission. In other instances, where either males or both sexes act as pests/vectors, the release of any sterile individuals has to be carefully managed, e.g. feeding tsetse males with trypanocidal drugs before their release or using strains with enhanced vector refractoriness (Kariithi et al. 2018). New methods are, therefore, needed that either completely remove any females from among the insects to be released, or reduce the ability of target species to act as pests/vectors, thus allowing the undesirable sex(es) to be tolerated among the sterile insects to be released.

\section{THE INCOMPATIBLE INSECT TECNIQUE (IIT)}

\subsection{Wolbachia, Cytoplasmic Incompatibility and Population Suppression}

The IIT is an analogue of the SIT, using infection with naturally-occurring maternallyinherited bacterial endosymbionts that cause reproductive parasitism - instead of radiation - to make released males reproductively incompatible with females of the target field population (Bourtzis et al. 2014; Scott and Benedict 2016; Xi and Joshi 2016). Wolbachia is the most common and widespread of such endosymbionts (Werren et al. 2008), being found throughout many insect orders, i.e. it is estimated to infect between approximately 48 to $57 \%$ of all terrestrial arthropods (Hilgenboecker et al. 2008; Zug and Hammerstein 2012; Weinert et al. 2015). One of the manipulations of host reproduction caused by Wolbachia is cytoplasmic incompatibility (CI), a form of conditional sterility whereby the fertilized eggs of females not infected with the same Wolbachia strain as the males with which they are mated, undergo embryonic death (Sinkins 2004; Werren et al. 2008; Hurst and Frost 2015). In contrast, Wolbachia-infected females produce off-spring normally, whether mated with uninfected males or with males infected with the same Wolbachia strain. 
The level of CI induced can vary considerably between Wolbachia strains: some strains do not cause CI - or any other reproductive manipulation - while others cause either partial or complete CI that either only kills some or all embryos, respectively. In general, Wolbachia and other similar endosymbiotic reproductive parasites are only maternally-inherited, but the level of transmission from mother-to-offspring can vary considerably. In mosquitoes, native Wolbachia infections typically exhibit very high levels $(\sim 100 \%)$ of both CI and maternal transmission (Sinkins 2004; Baton et al. 2013), while these characteristics are often markedly lower $(<50 \%)$ and more variable in other Diptera, such as well-studied drosophilids.

As CI prevents uninfected females - or those infected with a different incompatible Wolbachia strain - from having off-spring, infected females leave more off-spring. The consequence is that, over succeeding generations, uninfected females can be driven to extinction as the number of infected females increases, potentially resulting in complete replacement of the original uninfected host population with Wolbachia-infected individuals (Caspari and Watson 1959; Fine 1978). The speed and extent with which population replacement occurs, i.e. replacing the original uninfected host population with Wolbachia-infected individuals, primarily depends on the level of CI-induced and the rate of endosymbiont maternal transmission, as well as whether or not the endosymbiont has any fitness costs or benefits for its hosts. When CI is complete, maternal transmission is perfect, and the endosymbiont has no fitness costs, complete population replacement is expected, and, at least theoretically, is predicted to be very rapid: occurring in about 100 generations, from a very low $(\sim 1 \%)$ initial proportion. If the initial proportion is higher (e.g. $>10 \%)$, population replacement could occur in < 10 generations (Caspari and Watson 1959; Fine 1978).

When CI is partial, and/or maternal transmission imperfect, and/or there are fitness costs, endosymbiont-infected individuals either will go extinct, or will only partially if not completely replace uninfected individuals if they constitute a certain proportion of the host population, known as the invasion threshold of the endosymbiont. During the process of population replacement, the size of the uninfected part of the host population is reduced due to the inhibition of reproduction by uninfected females as a result of mating with incompatible infected males (Dobson et al. 2002a). This creates a positive feedback-loop that increases the relative proportion of infected individuals in the population, as well as creating vacant niche space to be filled, and thereby accelerates and drives the rate of both replacement of uninfected individuals and the degree of population suppression (as uninfected females are increasingly more likely to mate with infected males). It is this naturally-occurring mechanism of host population suppression that is exploited by IIT. However, as only males should be released during IIT application, the subsequent population replacement by endosymbiont-infected individuals that occurs in natural systems does not occur during target population suppression, as there are no infected females to maternallytransmit the endosymbiont to the next generation. The consequence is target population elimination.

An alternative strategy for vector control which is currently being extensively investigated and actively implemented - and which we do not discuss further here involves intentionally releasing endosymbiont-infected females in order to deliberately trigger population replacement (Sinkins et al. 1997; Iturbe-Ormaetxe et 
al. 2011; Bourtzis et al. 2014; Xi and Joshi 2016). As described in Section 3.4.2 below, some Wolbachia infections can reduce vector competence for vector-borne pathogens, such that population replacement with such endosymbiont variants would reduce or prevent pathogen transmission by a vector population. However, we regard the aim of population suppression as preferable to population replacement, because it can be guaranteed to completely prevent any future pathogen transmission (endosymbiontmediated reduction in vector competence may not be complete, and/or may be lost over time due to the evolution of resistance by the transmitted pathogen and/or changes in the vector-endosymbiont association), and is likely to have greater public acceptance due to male-only releases, the reduction of nuisance biting, and the possibility of vector eradication (Zheng et al. 2019).

\subsection{A Brief History of the IIT}

CI was first observed in the mosquito Culex pipiens L. (Marshall and Staley 1937; Marshall 1938; Roubaud 1941), shortly after the independent discovery of the endosymbiont Wolbachia in the same mosquito species (Hertig and Wolbach 1924; Hertig 1936). However, it was not until more than three decades later that the causal link between Wolbachia and CI was hypothesized, and then empirically proven through curing mosquitoes of their bacterially-induced CI by antibiotic treatment (Yen and Barr 1971, 1973).

The notion of using CI for suppression of vector populations was developed during the $1960 \mathrm{~s}$ - that is, prior to the realization that maternally-inherited endosymbionts cause CI - as part of a World Health Organization (WHO)-sponsored programme instigated and led by the German entomologist Hannes Laven (WHO 1964; Pal 1966; Knipling et al. 1968; Laven 1971; Davidson 1974). In unpublished studies, it was first shown, using cage experiments, that the release of incompatible males at an initial 1:1 ratio with target-compatible males, could eradicate a stable target population in only 3 or 4 generations (Pal 1966; Laven 1967, 1971).

Consequently, a small-scale open-release pilot trial in the field was undertaken in a relatively isolated rural village (Okpo) near Rangoon in Myanmar (Burma) against the local vector of filariasis, Culex quinquefasciatus Say (Laven 1967, 1971). This trial was a resounding success, effectively eliminating the local mosquito population by the end of the 12-week intervention period, although there were some reservations about the significance, and general applicability, of this "proof-of-principle" demonstration (Laird 1967; Barr 1970; Weidhaas and Seawright 1976).

Subsequently, a larger-scale joint WHO / Indian Council of Medical Research (ICMR)-backed project to further investigate the feasibility of using the IIT was established in the 1970s in India (Grover and Sharma 1974; Pal 1974), resulting in a number of studies characterizing the incompatibility, mating competitiveness and vector competence of endosymbiont-infected mosquitoes (Subbarao et al. 1974, 1977; Grover et al. 1976; Singh et al. 1976; Curtis 1977; Krishnamurthy 1977; Thomas and Singh 1977; Curtis and Reuben 2007), as well as the first attempts of combining the IIT with genetic modification (Laven and Aslamkhan 1970; Krishnamurthy and Laven 1976; Curtis 1977). The results of the field trials were less convincing than before with only partial population suppression $(<70 \%)$ achieved, apparently due to 
unexpected high levels of immigration of previously inseminated females from the areas surrounding the release sites (Brooks et al. 1976; Curtis 1977; Curtis et al. 1982).

During this latter period, with the discovery of CI in tephritid flies (the European cherry fruit fly Rhagoletis cerasi L., Boller and Bush 1974), and pyralid moths (the almond moth Cadra cautella (Walker), Brower 1976), there was interest for using CI to suppress other pest insects (Russ and Faber 1979; Neuenschwander et al. 1983; Blümel and Russ 1989; Boller 1989), with the term "IIT" being coined (Boller et al. 1976), and several promising laboratory studies and semi-field trials undertaken (Brower 1979, 1980; Ranner 1990).

During the 1980s, interest in the IIT (and the SIT) for mosquitoes waned (Scott and Benedict 2016), partly due to the premature termination of the joint WHO/ICMR project (Anonymous 1975; Curtis and Reuben 2007), but also because of doubts about the practical feasibility and economics of rearing large numbers of mosquitoes, as well as the possibility/sustainability of population suppression/elimination in the presence of immigration from outside control areas (Sinkins et al. 1997; Scott and Benedict 2016).

From the 1990s to the present, a new generation of researchers and their academic descendants have given fresh impetus to investigating the use of Wolbachia for pest and vector control (Iturbe-Ormaetxe et al. 2011; Bourtzis et al. 2014; Xi and Joshi 2016), resulting in a renewed interest in the IIT and its operational deployment (O'Connor et al. 2012; Mains et al. 2016, 2019; Zheng et al. 2019). The IIT has been under consideration for controlling the Mediterranean fruit fly Ceratitits capitata (Wiedemann) (Zabalou et al. 2004, 2009), the olive fruit fly Bactrocera oleae Rossi (Apostolaki et al. 2011), and the spotted wing drosophila Drosophila suzukii (Matsumura) (Cattel et al. 2018; Nikolouli et al. 2018), as well as tsetse flies (Glossina spp.) (Alam et al. 2011; Bourtzis et al. 2016). However, the development and implementation of the IIT was, and remains, the most advanced for mosquitoes, with open-release field trials planned or already recently undertaken for the arboviral and/or filarial vector species Aedes aegypti L. in Australia, Mexico, Singapore, and the USA (Xi and Manrique-Saide 2018; Yeung 2018; Corbel et al. 2019; Mains et al. 2019); Aedes albopictus (Skuse) in China and the USA (Mains et al. 2016; Zheng et al. 2019); and Aedes polynesiensis Marks in French Polynesia (Brelsfoard et al. 2008; O'Connor et al. 2012); as well as for Cx. quinquefasciatus on the four islands in the south-western Indian Ocean (La Réunion, Mauritius, Grande Glorieuse and Mayotte) (Atyame et al. 2011, 2015).

\subsection{Generating and Characterizing Novel Endosymbiont Infections}

In order to control a target pest or vector species using the IIT, it is necessary to have incompatible individuals for mass-rearing and release. The simplest method for obtaining such individuals is to collect them from the field. This is possible, for example, if different geographic populations of a target species naturally possess different incompatible endosymbiont infections (Brower 1976; Chen et al. 2013). This was the origin of the incompatible individuals used for the first IIT trials against $C x$. quinquefasciatus (Laven 1967, 1971), as well as other pest insects. However, there are several problems that require attention. 


\subsubsection{Introgressing the Nuclear Genome of the Target Population}

The first problem is that individuals from one geographic area may not be welladapted to another location and may have lower mating competitiveness compared to local males from the target population (Barr 1966). This problem can be solved by using backcrosses to introgress the nuclear genome of the target population into the incompatible colony to be used for releases (Barr 1966; Krishnamurthy 1977), which is achieved by mating males from the target population to females from the incompatible colony. The process is repeated but using the daughters of each cross instead. As Wolbachia and other endosymbiotic reproductive parasites are maternally inherited, the outcome is a new line that possesses the cytoplasmic organelles (mitochondria and endosymbionts) of the original incompatible line, but now with the nuclear genome of the target population.

This laborious technique is still used to create Wolbachia-infected lines (Atyame et al. 2011), and it remains a fundamental method for matching the genetic background of released individuals to the target population in the field. This technique was also used more recently to transfer through inter-specific introgression a naturally occurring Wolbachia infection into a target species (Ae. polynesiensis) from a closely related non-target sister species (Aedes riversi Bohart \& Ingram) (Brelsfoard et al. 2008).

\subsubsection{Generating Artificial Wolbachia Infections through Transinfection}

The second - and more significant - problem with relying on naturally-occurring endosymbiont infections is that it limits the availability and diversity of incompatible individuals, and, therefore, the insect species that are amenable to control using the IIT. Although endosymbionts are widespread among arthropods, many important pest and vector species, such as the mosquito Ae. aegypti, as well as the many mosquito species in the Anopheles genus of malaria vectors, are thought not to be naturally infected with Wolbachia (Bourtzis et al. 2014).

Even in those species that are infected with Wolbachia, there is often no intraspecific geographic variation in the endosymbionts and their mating compatibilities (as might be expected given the nature of their reproductive parasitism), and closelyrelated infected sister taxa capable of inter-specific interbreeding may not exist. The many intra-strain mating types observed in Cx. pipiens, which enable the IIT against this target species (Laven 1967; Atyame et al. 2011), are apparently atypical (Bourtzis et al. 2014). Although host species are not infrequently superinfected with two or more endosymbiont strains, these are often found throughout their geographic range (e.g. Ae. albopictus) (Bourtzis et al. 2014).

The ability to generate artificial Wolbachia infections in the laboratory through transinfection between individuals within the same or different host species was, therefore, a major breakthrough (Boyle et al. 1993; Braig et al. 1994). The application of these techniques has since provided renewed impetus to the use of endosymbionts for pest and vector control (Xi et al. 2005b; Hughes and Rasgon 2014), enabling the first open-release in the field of artificially-transinfected Wolbachia-infected mosquitoes for IIT application (Mains et al. 2016). 
For mosquitoes, transinfection can be achieved either through embryonic (Xi and Dobson 2005) or intra-thoracic microinjection of adults (Ruang-areerate and Kittayapong 2006). However, in order to establish stable germline infections, the former method is regarded as the most efficient, due to the low likelihood of the somatic infections resulting from the latter colonizing the gonads and being maternally transmitted (Hughes and Rasgon 2014). For other insects, inoculation of larval or pupal stages has also been occasionally reported, as has transfer through corearing and predation.

Xi et al. (2005b) successfully established the first artificial Wolbachia infection of mosquitoes through embryonic microinjection of the cytoplasm from endosymbiontinfected donor eggs (Xi and Dobson 2005), and since then, a number of different artificial germline Wolbachia infections have been established in mosquitoes, including Ae. aegypti (Xi et al. 2005b; McMeniman et al. 2009; Walker et al. 2011; Ant and Sinkins 2018), Ae. albopictus (Xi et al. 2005a; Xi et al. 2006; Suh et al. 2009; Calvitti et al. 2010; Fu et al. 2010; Blagrove et al. 2012; Ant and Sinkins 2018; Moretti et al. 2018b; Zheng et al. 2019), and Ae. polynesiensis (Andrews et al. 2012), as well as the malaria vector Anopheles stephensi Liston (Bian et al. 2013).

Artificial transfer of CI-inducing Wolbachia has now also been achieved in a number of different insect groups, including other Diptera (Drosophilidae and Tephritidae), as well as Lepidoptera, Hemiptera and Coleoptera (Hughes and Rasgon 2014).

Transinfection allows both naturally uninfected, as well as already infected, target species to be artificially infected with Wolbachia. In the latter instance, pre-existing native endosymbiont infections can either be first removed by antibiotics or moderately high temperature (Yen and Barr 1973; Portaro and Barr 1975; Dobson and Rattanadechakul 2001), and then replaced with a different strain of Wolbachia (Suh et al. 2009; Calvitti et al. 2010; Andrews et al. 2012).

Alternatively, novel superinfections can be generated by adding new artificial infestations of Wolbachia strains to the endosymbiont strains already present in the target populations (Fu et al. 2010; Joubert et al. 2016; Ant and Sinkins 2018; Zheng et al. 2019). Establishing superinfections (especially triple infections) may be trickier than replacing pre-existing endosymbiont infections (due to competitive interactions and/or incompatibilities between different Wolbachia strains, e.g. Ant and Sinkins 2018), but come with the added benefit of higher endosymbiont densities and broader somatic tissue distributions, which is thought to be of importance for altering pest/vector status (see Section 3.4.2) (Moretti et al. 2018b; Zheng et al. 2019), but not necessarily for the induction of CI.

\subsubsection{Selection of Endosymbionts for Transinfection}

The potentially unconstrained ability to transfer any Wolbachia strain between any host species raises the issue of selecting which endosymbiont strains to transinfect (Hoffmann et al. 2015). So far, only a relatively limited number of Wolbachia strains from well-studied hosts have been tried (Hughes and Rasgon 2014), but Wolbachia is an ancient, phenotypically diverse, and vast bacterial clade spread across phylogenetically-distant host taxa, with potentially more strains (i.e. millions) than infected host species (due to the occurrence of superinfection) (Werren et al. 2008). 
Although the characteristics of endosymbionts after host transfer can sometimes be unpredictable, and depend upon host background (Hoffmann et al. 2015), the behaviour of a given Wolbachia strain in one host generally provides a reasonable "rule-of-thumb" for predicting its behaviour in other hosts, especially if those hosts are phylogenetically-related, enabling some guidance in the selection of endosymbiont strains to be transferred.

For example, the unusually virulent Wolbachia strain wMelPop (Min and Benzer 1997), generally retains its pathogenicity, whether present in closely or more distantly related dipteran hosts. Similarly, native Wolbachia infections in mosquito species generally have the same characteristics when transferred to new mosquito hosts (see below).

An important exception to this pattern seems to be that novel Wolbachia infections often have higher endosymbiont strain-specific densities and/or broader somatic tissue distributions, which are associated with host fitness and other phenotypic effects (Hoffmann et al. 2015; Xi and Joshi 2016; Ant and Sinkins 2018).

\subsubsection{Characterization of New Host-endosymbiont Associations}

Once endosymbiont-infected individuals have been found from the field or generated de novo in the laboratory, their host-endosymbiont association needs to be thoroughly characterized to determine if it is suitable for IIT application. The basic requirements for an endosymbiont to be used for the IIT are to induce CI, have favourable levels of maternal transmission, and, in general, to have low fitness costs. CI is required to generate the male incompatibility that enables sterilisation of wild-type females in the target population. Stable maternal transmission is required to ensure that males can cause CI, and to enable their efficient mass-production.

If males are not infected, they cannot induce CI, and if maternal transmission is low then many uninfected individuals will be produced in each generation. As there is currently no method to separate the uninfected from the infected individuals, their presence during factory rearing requires more individuals to be mass-produced, and more males to be released, for a given level of target population suppression. In addition, if maternal transmission is unstable, it can result in self-incompatibility between superinfected individuals, compromising colony maintenance and preventing mass-production of appropriately infected individuals (Ant and Sinkins 2018).

Low fitness costs of Wolbachia infection are required to enable efficient massproduction of large numbers of factory-reared individuals for release, as well as to ensure the mating competitiveness of the released males.

Many, although not all, of the artificially infected mosquito lines have been shown to have these characteristics, inducing high levels $(\sim 100 \%)$ of $\mathrm{CI}$, when the transinfected males mate with wild-type females, causing high levels $(\sim 100 \%)$ of stable maternal inheritance, and having no or only low fitness costs (Section 3.4.1) (Xi et al. 2005b; Bian et al. 2010, 2013; Calvitti et al. 2010, 2012; Blagrove et al. 2012, 2013; Joshi et al. 2014; Zheng et al. 2019). 


\subsection{Advantages of Using Endosymbionts}

The fundamental difference between the IIT and the SIT is the sterilizing procedure: infection with CI-inducing endosymbionts in the former, and irradiation in the latter (Bourtzis and Robinson 2006). Other aspects of the IIT and the SIT tend to be common to all sterile-male-based methods (Alphey et al. 2010; Bourtzis et al. 2016; Dyck et al. 2021), although the use of endosymbionts entails some specific considerations (Section 5).

As discussed above (Section 2.1), there are potentially direct and/or indirect harmful effects associated with irradiation-based sterilisation, which can both be circumvented by using CI-inducing endosymbionts. Although the initial introduction of a novel endosymbiont strain into a target species is not trivial, requiring considerably more effort, time and specialist skill than administering a single dose of irradiation (Hughes and Rasgon 2014), it only needs to be done once. As CI-inducing endosymbionts are maternally-inherited, once stably introduced into the germline of a target species, incompatibility is self-perpetuating and maintained across generations, so that there is no need for repeated rounds of sterilisation - with their associated economic and biological costs - within and across generations, as is the case for irradiation-based sterilisation. The use of radiation also entails various logistical and bureaucratic requirements (e.g. infrastructure and regulatory frameworks), which are not necessary when using endosymbiont infection. In addition, use of CI-inducing endosymbionts allows greater flexibility with regard to the life cycle stages of the target species that can be released (Bourtzis and Robinson 2006), while the SIT is often restricted by the life cycle stage at which irradiation is optimally performed.

Another overlooked advantage of the IIT is that the released individuals are conveniently "tagged" by their endosymbiont infections: there is no need to additionally mark released insects using chemical dyes - which may impose fitness costs (Curtis et al. 1982) - in order to track them during control programmes (Bourtzis and Robinson 2006). Identification of infected or sterile males, or their sperm, can be done by PCR (O'Connor et al. 2012; Juan-Blasco et al. 2013; Mains et al. 2016, 2019; Zheng et al. 2019). In addition, Wolbachia may have beneficial effects on larval development, such as promoting faster development, and thus lower rearing costs (Zhang et al. 2015a; Puggioli et al. 2016).

Some potential disadvantages of using endosymbionts, other than the major one of accidental female release resulting in unintended population replacement (see Section 3.5), are that incompatibility may decline with increasing adult male age (Tortosa et al. 2010), and with male sperm depletion following multiple mating (Bourtzis and Robinson 2006). So far, the decline in male incompatibility with age, which occurs with some native Wolbachia infections (Singh et al. 1976; Krishnamurthy et al. 1977; Calvitti et al. 2015), has not been reported for artificial infections (Moretti and Calvitti 2013), possibly due to the higher endosymbiont densities of the latter (Calvitti et al. 2015). Sperm depletion also affects the SIT, and, again, may occur during native Wolbachia infections, but it has been reported to have no effect for artificial endosymbiont infections (Turley et al. 2013). 


\subsubsection{Cytoplasmic Incompatibility without Male Fitness Costs}

A widely-perceived advantage of the male incompatibility caused by CI-inducing endosymbionts, such as Wolbachia, is that it often has either no or only minor effects on male quality (Pal 1966; Laven 1974; Boller et al. 1976; Brower 1976; Sinkins et al. 1997; Scott and Benedict 2016). Although Wolbachia infections can be highly virulent (Min and Benzer 1997; McMeniman et al. 2009; Suh et al. 2009; Rasgon 2012), this is apparently atypical. In general, in their co-evolved native hosts, maintained under field conditions, these endosymbionts are thought to more commonly reside in the commensal to mutualist region of the spectrum of symbiosis (if their parasitic and "spiteful" reproductive manipulations are not considered) (Xi and Joshi 2016). CI-inducing endosymbionts can be expected to have been optimized over many millennia of natural selection to specifically induce sterility, while minimizing any harmful effects on male quality, as this would reduce their capacity to invade host populations (Segoli et al. 2014).

Consistent with this theoretical understanding, native Wolbachia infections of mosquitoes have generally been reported to have no effects on male quality (Dobson et al. 2002b; Calvitti et al. 2009; Baton et al. 2013). Although several studies have reported reduced mating competitiveness of field released incompatible males ( $\sim 30$ to $70 \%$ ) (possibly due to the possession of a sterility-inducing chromosomal translocation in one study, and the use of chemical marker dyes in another) (Grover et al. 1976; O'Connor et al. 2012), the majority of studies have shown both native and artificially infected males to have mating competitiveness equal to that of wild-type males in both laboratory and field settings (Brower 1978; Curtis et al. 1982; Arunachalam and Curtis 1985; Blagrove et al. 2013; Moretti and Calvitti 2013; Joshi et al. 2014; Segoli et al. 2014; Atyame et al. 2015; Axford et al. 2016; Puggioli et al. 2016; Zhang et al. 2016; Zheng et al. 2019), with some even suggesting increased mating competitiveness for incompatible males (Puggioli et al. 2016; Moretti et al. 2018b).

In comparison to other aspects of endosymbiotic reproductive parasites, the effects of CI-inducing endosymbionts on the individual components of male fitness, such as sperm competition, are relatively under-studied, although the highly virulent $w$ MelPop strain has been found to have no effect on insemination rates, or sperm quantity and viability (Turley et al. 2013). However, the mating competitiveness studies described above imply that the individual components of male fitness are generally unaffected by Wolbachia infection.

Many studies have found that Wolbachia infection has no effect on male longevity, while some studies have even found that artificial Wolbachia infections significantly increase adult male survival, which might increase the efficiency of incompatible males in IIT programmes (Blagrove et al. 2013; Joshi et al. 2014). A few studies have also compared irradiation-based sterilisation with endosymbiont-induced incompatibility, but these have not always used the most appropriate comparison (i.e. uninfected and irradiated individuals compared to non-irradiated Wolbachia-infected individuals with the same genetic background) (Atyame et al. 2016; Puggioli et al. 2016; Zhang et al. 2016; Zheng et al. 2019). In addition, standardized protocols need to be developed to enable robust comparison between these different sterilisation methods (Bourtzis et al. 2016). 


\subsubsection{Other Useful Phenotypes Enabling Tolerance of the Undesired Sex(es)}

In addition to causing incompatibility, endosymbiotic reproductive parasites like Wolbachia can also have a range of other phenotypic effects on their invertebrate hosts, including effects on both adult male and female fitness. One of the most important phenotypic effects is that Wolbachia can inhibit viral pathogens (Hedges et al. 2008; Teixeira et al. 2008), and artificially-transinfected mosquitoes have been shown to often strongly inhibit or completely block a variety of vector-borne pathogens, especially arboviruses, including dengue, chikungunya, Mayaro, West Nile, yellow fever, and Zika viruses, and to a lesser extent filaria and malaria parasites (Kambris et al. 2009; Moreira et al. 2009; Bian et al. 2010, 2013; Glaser and Meola 2010; Andrews et al. 2012; van den Hurk et al. 2012; Aliota et al. 2016; Dutra et al. 2016; Joshi et al. 2017; Pereira et al. 2018; Zheng et al. 2019). As well as direct effects on pathogen infection, endosymbionts might also indirectly reduce pathogen transmission, for example by reducing the survival of their adult female vectors (Brownstein et al. 2003; Rasgon et al. 2003; Cook et al. 2008).

In contrast, native Wolbachia infections tend to have less predictable effects on vector-borne pathogens and have been reported to inhibit, enhance or have no effect upon them (Curtis et al. 1983; Dutton and Sinkins 2005; Bian et al. 2010; Blagrove et al. 2012; Graham et al. 2012; Baton et al. 2013; Bourtzis et al. 2014; Zélé et al. 2014). If possible, any released insects should be lower pests/vectors than the target population (Laven and Aslamkhan 1970; Thomas and Singh 1977). Given the potentially variable effects of endosymbiont infection on pest/vector status, this aspect of target species biology should be thoroughly characterized prior to releasing Wolbachia-infected insects (e.g. Zheng et al. 2019).

The ability of Wolbachia to reduce the ability of target species to act as pests or vectors, enables the release of the sex(es) which are pests/vectors to be tolerated, compensating for imperfect sex separation (Section 2.2) (Moretti et al. 2018b; Zheng et al. 2019), and could be an additional means to make the release of male pests/vectors more tolerable (e.g. tsetse flies) (Bourtzis et al. 2016; Kariithi et al. 2018). The ability of CI-inducing endosymbionts to reduce the ability of insects to act as pests/vectors also provides an important fail-safe during IIT implementation, given the high probability of accidental female release during operational programmes (Section 3.5), as unintended population replacement would then reduce pathogen transmission, while target population suppression would not be achieved (Zheng et al. 2019).

\subsection{The Problem of Unintended Population Replacement}

An inherent and significant problem with the IIT, in the absence of perfect sex separation methods, which has been recognized since the idea of IIT use was first conceived, and has so far prevented its operational use, is that, unlike SIT, the accidental release of Wolbachia-infected females may prevent further population suppression by causing unintended population replacement (Barr 1966; Pal 1974; Curtis 1977). 
When the IIT is deployed, release of incompatible males will prevent reproduction by the wild-type females of the target population, and the target population will become suppressed. However, if any Wolbachia-infected females are accidentally released along with the released males, the former may successfully begin to reproduce in the field, because they are compatible with the released males infected with same Wolbachia strain, as well as the wild-type males in the original target population. Consequently, after an initial period of population suppression, during which the reproduction of wild-type females is inhibited, the original target population may become replaced by a population with Wolbachia-infected individuals (Section 3.1).

Whether population replacement actually occurs will depend upon a number of parameters, such as the number of females accidentally released, and the characteristics of the Wolbachia infection used to make released males incompatible with the target population. In principle, a single female could trigger population replacement, if her Wolbachia infection causes high levels of CI, has high maternal transmission, and no fitness costs (Section 3.1). In reality, stochastic and population density-dependent processes mean that a single female is unlikely to leave surviving off-spring, even if her Wolbachia infection has no invasion threshold. Just how many released females are required to inevitably trigger population replacement is unknown, and difficult to quantify accurately in the absence of relevant empirical data. However, the risk of population replacement will clearly increase as the original uninfected target population is suppressed, because this will inevitably increase the relative proportion of any accidentally-released Wolbachia-infected females (and their descendants) in relation to the wild-type individuals of the original target population, increasing the likelihood that the former passes its invasion threshold (Section 3.1).

Furthermore, if the original target population is eliminated, rather than merely suppressed, there will be a vacant niche that will be filled, by default, by any accidentally released females (Curtis 1977), which are now more likely to be able to establish a field population, as any inhibitory stochastic and density-dependent processes related to intra-specific competition with wild-type uninfected individuals will now be relaxed (Berryman et al. 1973; Weidhaas and Seawright 1976; Dobson et al. 2002b). Whether population replacement occurs will then mostly depend on the characteristics of the Wolbachia infection used to induce incompatibility (i.e. the Wolbachia-infected individuals might go extinct if the Wolbachia infection imposes severe fitness costs, but otherwise they would be expected to persist, given the general characteristics of the Wolbachia strains so far used for IIT application - see Sections 3.1 and 3.4).

Laboratory cage experiments and mathematical modelling both indicate that inundative releases of incompatible males, contaminated with some females, facilitates Wolbachia invasion and population replacement (Hancock et al. 2011; Bian et al. 2013; Moretti et al. 2018a; Zheng et al. 2019). In addition, artificial Wolbachia infections, with the requisite characteristics, have been able to invade and persist in field populations (Hoffmann et al. 2011, 2014). These observations reinforce the notion that the risk of unintended population replacement following accidental female release is real, and not merely a "hypothetical" or purely academic concern. 
Some researchers have claimed that the risk of unintended population replacement can be minimized by releasing Wolbachia-infected insects that are bidirectionally incompatible with their wild-type target field population (Sharma et al. 1979; Calvitti et al. 2012; Bourtzis et al. 2014). Bidirectional CI occurs when the target population is infected with its own native Wolbachia strain, which causes incompatibility when wild-type field males mate with released females infected with a different Wolbachia strain that is used for IIT. The reproduction of any accidentally released females, therefore, will be prevented if they mate with the wild-type field males. This contrasts with unidirectional CI, which occurs when the target field population is either uninfected or infected with Wolbachia strain(s) that are not incompatible with the Wolbachia strain used for IIT, enabling any accidentally released females to successfully reproduce when mated wild-type field males.

However, explicit mathematical modelling and laboratory cage experiments indicate that bidirectional CI will only provide protection against population replacement if the frequency of the released Wolbachia strain does not exceed its own invasion threshold (Dobson et al. 2002a; Moretti et al. 2018a). Although this is unlikely to happen for bidirectional CI at low or intermediate levels of target population suppression, this is not, in general, the intended endpoint of sterile-malebased methods, which aim for high levels of population suppression, if not population elimination, at which point any accidentally-released bidirectionally incompatible females will exceed their invasion threshold. In practice, therefore, bidirectional CI does not provide appreciably greater protection from population replacement than unidirectional CI, if the aim is either population elimination or merely to reduce target population densities below that which causes a pest/vector problem (i.e. high levels of population suppression).

In addition, any released residual females are much more likely to mate with the males with which they are released, than with males in the field population (as the former are held together overnight in containers before their release, and afterwards released males vastly outnumber those in the field) (Zheng et al. 2019). Thus, the advantage from incompatible matings between released residual females and field males due to bi-directional CI may be negligible from a practical standing point.

Although several small-scale short-term field trials have reported the use of the IIT without the apparent consequence of population replacement (O'Connor et al. 2012; Mains et al. 2016, 2019; Zheng et al. 2019), it should be noted that in none of these instances was the target population sufficiently suppressed to enable the rapid replacement by any released Wolbachia-infected individuals which might occur as population elimination is approached (i.e. within the $\sim 6$ month time-scale of the reported results of these field trials). These studies also involved the release of relatively small numbers of mosquitoes using manual sex separation, which ensured lower female contamination rates than when using mechanical sex separation. However, manual separation is impractical for medium- to large-scale area-wide applications (Pal 1974; Brelsfoard et al. 2008; Zheng et al. 2019). As such, the above described small-scale field trials were probably unlikely to have released enough Wolbachia-infected females to enable establishment of Wolbachia-infected field populations. 


\section{COMBINED IIT/SIT APPLICATION}

\subsection{The Solution to an "Intractable" Problem}

In order to solve the inevitable problem of accidental female release, the second series of IIT field trials undertaken in India during the 1970s (Section 3.2) combined the IIT with genetic modification of the released insects, such that they carried a chromosomal translocation that induced semi-sterility when they mated amongst themselves (Laven and Aslamkhan 1970; Brooks et al. 1976; Krishnamurthy and Laven 1976; Curtis 1977; Curtis et al. 1982). Although this approach seemed to show promise in the laboratory, under field conditions it had little impact.

As an alternative, Curtis (1977), who described the problem of unintended population replacement following accidental female release during IIT implementation as "intractable", proposed, nonetheless, a practical, and, as it turns out, viable solution: combined IIT/SIT use. His solution was to exploit the fact that female insects are often more sensitive than males to radiation (Bakri et al. 2005, 2021), and to combine the IIT with the SIT, such that all incompatible individuals destined for field release were first subjected to low-dose radiation, which would completely sterilize any contaminant females without affecting the incompatibility or quality of the simultaneously irradiated males. The combination of the SIT and the IIT for mosquito control is shown in Fig. 1. Although some preliminary laboratory investigations were undertaken by Curtis and others (Sharma et al. 1979; Arunachalam and Curtis 1985; Shahid and Curtis 1987), the notion of using the combined IIT/SIT was neglected for several decades until its re-assessment in more recent times (Bourtzis and Robinson 2006; Brelsfoard et al. 2009; Zhang et al. 2015b, 2016; Kittayapong et al. 2018, 2019; Zheng et al. 2019; Kittayapong, this volume; Liew et al., this volume).

\subsection{Optimum Irradiation Dose for Female Sterilisation}

Combined IIT/SIT application requires that females are more sensitive to the effects of irradiation than males, and that this difference is sufficiently large to enable complete sterilisation of females without appreciably or only minimally impacting male quality (Curtis 1977). If this inherent biological condition is met, it is necessary to determine the optimum irradiation dose. At first thought, this might be considered to be the minimum irradiation dose required to completely sterilize females, under the assumption that higher doses of radiation would begin to negatively affect males, and thereby undermine the main rationale for using endosymbiont-induced CI.

Accordingly, a number of preliminary studies have characterized the relative susceptibility of females and males to irradiation, with the aim of identifying the minimum irradiation dose required to completely sterilize females (Sharma et al. 1979; Shahid and Curtis 1987; Arunachalam and Curtis 1985; Brelsfoard et al. 2009; Zhang et al. 2015b, 2016). These irradiation studies showed that, at least for mosquitoes, females are indeed more sensitive than males to irradiation, and that there are levels of irradiation that can completely sterilize females, without appreciably impacting on male quality. Importantly, these and other studies have also shown that 
the irradiation treatment used to completely sterilize females has no effect on the level of CI induction by the co-irradiated Wolbachia-infected Ae. albopictus males (Zheng et al. 2019).

A

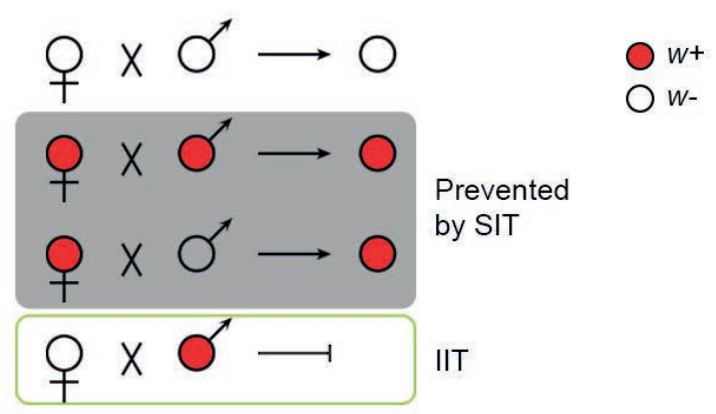

B

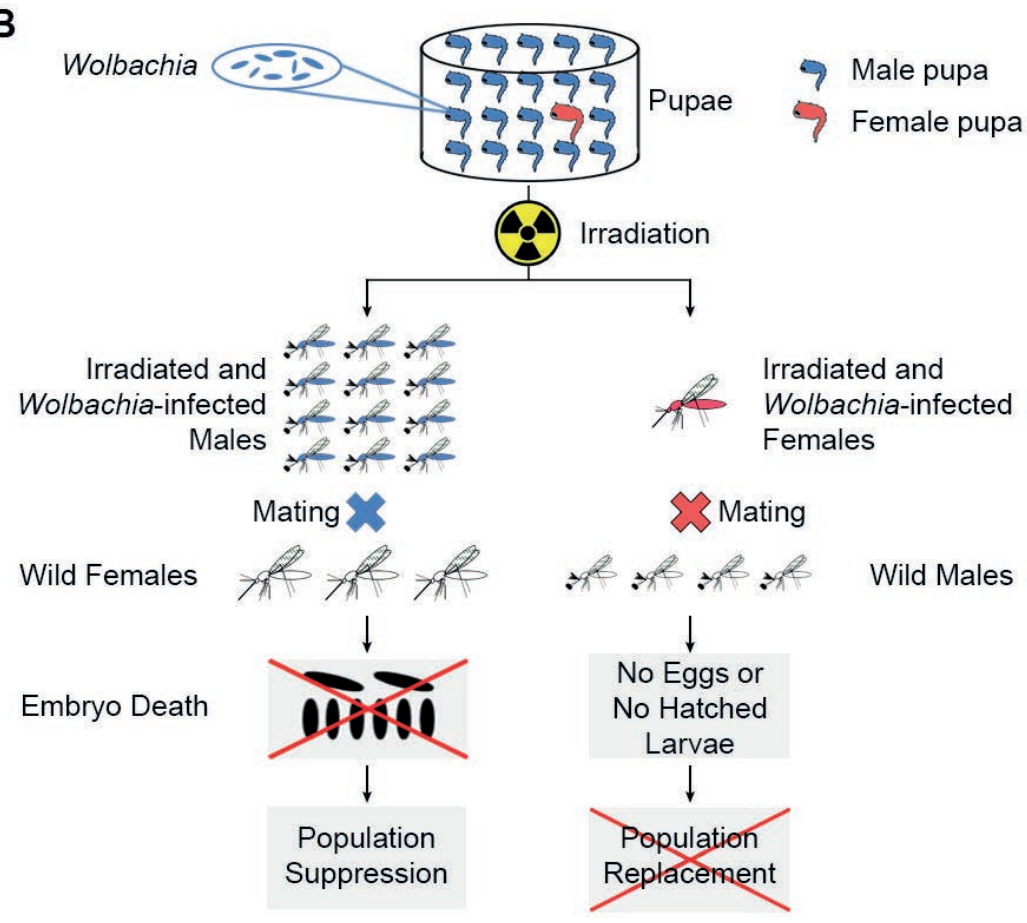

Figure 1. Schematic diagrams illustrating the combined IIT/SIT approach. (A) The four different types of crosses possible between wild-type uninfected and wPip-infected Aedes albopictus, and the role of irradiation in prevention of residual infected females from reproducing in the field. Red indicates Wolbachia-infected individuals $(\mathrm{W}+)$, while white indicates uninfected individuals (W-). (B) Illustration of production, irradiation, and release of Wolbachia-infected males with the residual females, respectively, and their mating with the wild population in the field. 
The optimal irradiation dose for the deployment of the combined IIT/SIT approach, however, is not necessarily the minimum irradiation dose for "complete" female sterilisation observed in laboratory studies, as the latter use small sample sizes, which defines a minimum detectable level of sterility, and different irradiation protocols (in particular, where there is no necessity to overcrowd pupae, as required during mass-irradiation, which may induce radio-protective hypoxia) (Yamada et al. 2019). It is vital for the IIT that any released females have no residual fertility, as this could render any current and future implementations using the same endosymbiont strain ineffectual. Consequently, combined IIT/SIT field releases involving millions of individuals need somewhat higher doses of irradiation than required in small-scale laboratory studies to ensure that all released females are fully sterilized (Yamada et al. 2019).

\subsection{Sequential IIT/SIT Application}

An alternative strategy related to the combined IIT/SIT releases to prevent population replacement resulting from accidental female release is a "sequential IIT/SIT" approach. This would involve initial IIT only releases, followed by SIT only releases (as opposed to the simultaneous combined IIT/SIT application in the same released individuals) (Atyame et al. 2016). The rationale here is that if the IIT is more efficient than the SIT because of the higher mating competitiveness and higher induced sterility of endosymbiont-infected males, large-scale only IIT releases can be used initially to suppress the target population, followed immediately by smaller-scale only SIT releases to eradicate - "mop-up" - any endosymbiont-infected individuals resulting from females inadvertently released during the initial phase of the IIT.

Whether a sequential IIT/SIT approach is preferable or superior to the combined IIT/SIT approach is not obvious and requires a careful quantitative comparison of the relative costs and benefits of the two strategies. Sequential IIT/SIT releases have the advantage that the males released during IIT application are not irradiated, maximizing their mating competitiveness, and removing the logistical costs/difficulties associated with large-scale irradiation (e.g. reduced male quality because of increased handling, etc.). However, these benefits would come with an increased risk of triggering population replacement in the first place (as many fertile females might now be released), the requirement to more carefully and rigorously monitor the target population to identify when/if population replacement occurs, and the risk of missing the optimal time window to switch to the SIT only releases (such that large-scale releases of relatively inefficient SIT, or other methods, would then be required).

Sequential IIT/SIT releases may be more convenient and effective in highly localized short-term programmes against geographically-restricted and lowabundance target populations, where, overall, relatively few incompatible males need to be initially released, and therefore the risk of accidental female release is inherently lower, while the combined IIT/SIT is likely to be more appropriate under the opposite conditions (i.e. area-wide long-term programmes against geographically-widespread and high-abundance target populations). 
When sequential IIT/SIT releases are used, if irradiation-based sterilisation of any females released during the SIT step is not complete, then these individuals could also establish a field population. For this reason, it might be prudent not to use for the SIT releases the same endosymbiont-infected insect line used for the initial IIT releases, so as to maintain the effectiveness of the initial insect line originally used for IIT should it be required for this latter purpose again (i.e. in multiple alternate rounds of IIT and SIT application).

In a similar manner to that envisaged for the sequential IIT/SIT, only SIT releases could also be used as a fail-safe after combined IIT/SIT application, should the latter fail to prevent population replacement.

\section{THE FIRST OPEN-RELEASE FIELD TRIAL OF COMBINED IIT/SIT}

Despite the previous exploratory laboratory studies determining the possibility and optimal dose for differentially sterilizing females and males for use in the combined IIT/SIT approach (Section 4.2.), there had been no previous experimental or field evaluation of this combined strategy. Consequently, a project was initiated, involving collaboration between Sun Yat-sen University, Michigan State University and other partners, to develop and field test combined IIT/SIT releases against the important mosquito arboviral vector Ae. albopictus.

This project involved a series of stages (Figs. 2 to 4), as described below, including initial laboratory studies to generate and then characterize an incompatible artificiallyWolbachia-infected Ae. albopictus line, subsequent "proof-of-concept" semi-field trials of the combined IIT/SIT approach, and then finally an open-field trial to demonstrate the feasibility of area-wide application of combined IIT/SIT releases for the management of an Ae. albopictus population.

\subsection{Generation and Characterization of Novel Wolbachia Infection}

The first requirement was to create a novel Wolbachia infection in Ae. albopictus that would generate incompatibility with wild-type males in our study area. To do this, the Wolbachia strain $w$ Pip was transferred by embryonic microinjection (Fig. 2) from its native mosquito host $C x$. pipiens into Ae. albopictus, to generate the new mosquito line HC. This line had a similar nuclear genetic background to individuals from the area of our field trial in Guangzhou, China, but in addition a novel triple Wolbachia infection (the artificially-transinfected $w$ Pip plus its two native Wolbachia strains) (Zheng et al. 2019). wPip was chosen because in its native mosquito host it has characteristics appropriate for IIT: it causes complete CI, has perfect maternal transmission, and no appreciable fitness costs. Indeed, upon transfer to its new host, these properties were retained. 


\section{Mosquito line generation and characterization}

Embryonic microinjection to generate one Wolbachia-infected female as a "seed" for mass rearing

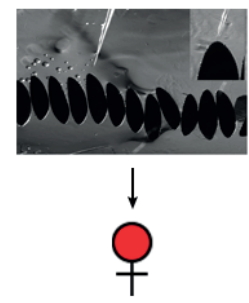

2. Mosquito mass rearing

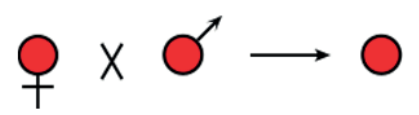

3. Field release

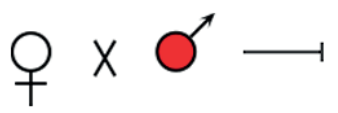

4. Field monitoring

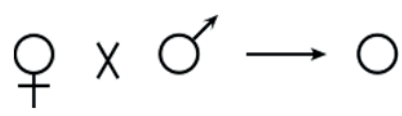

Figure 2. Overview of the IIT or combined IIT/SIT approach. First, a mosquito line with the novel Wolbachia infection is generated by embryonic microinjection. The hallmark of success

in this step is the generation of an infected female with $\sim 100 \%$ maternal transmission efficiency to pass Wolbachia into their offspring (2.1). Then, the infected individuals are mass-produced in the factory (2.2), or also irradiated. The infected males are subsequently released into the field to induce sterility in the wild population (2.3). The density of the wildtype uninfected population is monitored to measure the effect of population suppression (2.4).

Red indicates individuals carrying the novel Wolbachia infection, while white represents wild-type individuals.

In laboratory studies, $\mathrm{HC}$ males caused complete CI when mated with wild-type females and had perfect maternal transmission (Zheng et al. 2019). There were also no differences between $\mathrm{HC}$ and wild-type Ae. albopictus in fecundity (number of eggs laid), fertility (egg hatch), larval/ pupal/ adult male or female survival, sex ratio or body size, although $\mathrm{HC}$ had a slightly faster larval development and adult emergence times (Zhang et al. 2015a). In addition, female HC had higher Wolbachia densities than wild-type females, and lower susceptibility to dengue and Zika virus infection, with both horizontal and vertical transmission of these arboviruses significantly reduced (Zheng et al. 2019). Although target population suppression/elimination was the aim of our field trial, the reduced vector competence of HC provided an important fail-safe should accidental female release and subsequently population replacement have occurred (Section 3.4.2) (Zheng et al. 2019).

\subsection{Laboratory and Semi-field IIT/SIT Trials}

A series of experimental laboratory studies were undertaken to characterize the HCline further, and to confirm that it could be used for combined IIT/SIT releases. Laboratory cage experiments indicated that $\mathrm{HC}$ male mating competitiveness was equal to that of wild-type males (Zhang et al. 2016; Zheng et al. 2019). They also showed that HC females could cause population replacement, when seeded into cages containing wild-type individuals, with the speed of this population replacement being enhanced when excess HC males (4:1 ratio with wild-type males) were 
simultaneously released (Zheng et al. 2019). As these experiments demonstrated the potential of accidentally released $\mathrm{HC}$ females to trigger population replacement during IIT application, the minimum irradiation dose (28 Gy) necessary to completely sterilize HC females was identified. This dose caused extensive damage to the ovaries, and hence prevented egg-laying as well as the establishment of $w$ Pip-infected individuals in a small laboratory cage population (Zhang et al. 2015b). This irradiation dose also did not affect mating competitiveness or survival of $\mathrm{HC}$ males, nor did it reduce CI induction (Zhang et al. 2016).

A subsequent semi-field trial was undertaken in field cages, which simulated the accidental release of $\mathrm{HC}$ females, in order to provide the first "proof-of-concept" that combined IIT/SIT application could prevent unintended population replacement (Zheng et al. 2019). Replicate control and experimental wild-type populations were established in large cages, into which irradiated excess HC males were repeatedly released (5:1 ratio with wild-type males), each time together with sufficient irradiated $\mathrm{HC}$ females to mimic a $2.0 \%$ contamination rate of the released $\mathrm{HC}$ males. Successful eradication of the wild-type populations occurred in all three of the field cages, without the occurrence of population replacement by the released $w$ Pip-infected HC mosquitoes, demonstrating that the combined IIT/SIT strategy works. Having demonstrated experimentally that the combined approach works, an open-release field trial was implemented in Guangzhou, China.

\subsection{Mass-production for Field Release}

In order to produce sufficient numbers of irradiated-incompatible males for openrelease during our combined SIT/IIT field trial, it was necessary to optimize rearing protocols and to develop new equipment to enable factory-scale mass-rearing and pupal irradiation (Fig. 3). Artificially-Wolbachia-infected mosquitoes do not require special rearing conditions and can be reared using the same protocols as those used for uninfected/wild-type individuals. However, some care should be given to ensure that Wolbachia-infected mosquitoes are not exposed to high temperatures or antibiotics (e.g. via their larval food or adult blood meals), as this could potentially remove their endosymbionts. In addition, larval rearing conditions may affect Wolbachia density, and, hence, possibly the level of CI expression, as well as maternal transmission of the endosymbiont (Puggioli et al. 2016). Consequently, it should be confirmed during the early stages of mass-production that the rearing conditions used do not adversely affect either the reproductive incompatibility or the quality of the males produced (Zhang et al. 2017, 2018).

For mass-rearing of larval mosquitoes, many rearing trays are required, thus several units for holding and storing large numbers of trays in order to improve space utilization have been developed at the FAO/IAEA Insect Pest Control Laboratory (IPCL) in Seibersdorf, Austria, and at the Wolbaki Institute of Biological Sciences in Guangzhou, China (Balestrino et al. 2012, 2014a; Zhang et al. 2017). The first generation "Wol-unit" holds 40 larval rearing trays, while only occupying $0.68 \mathrm{~m}^{2}$ of floor space, and enables simultaneous rearing of 264000 larvae, generating up to 89000 male pupae per rearing cycle (Zhang et al. 2017). 

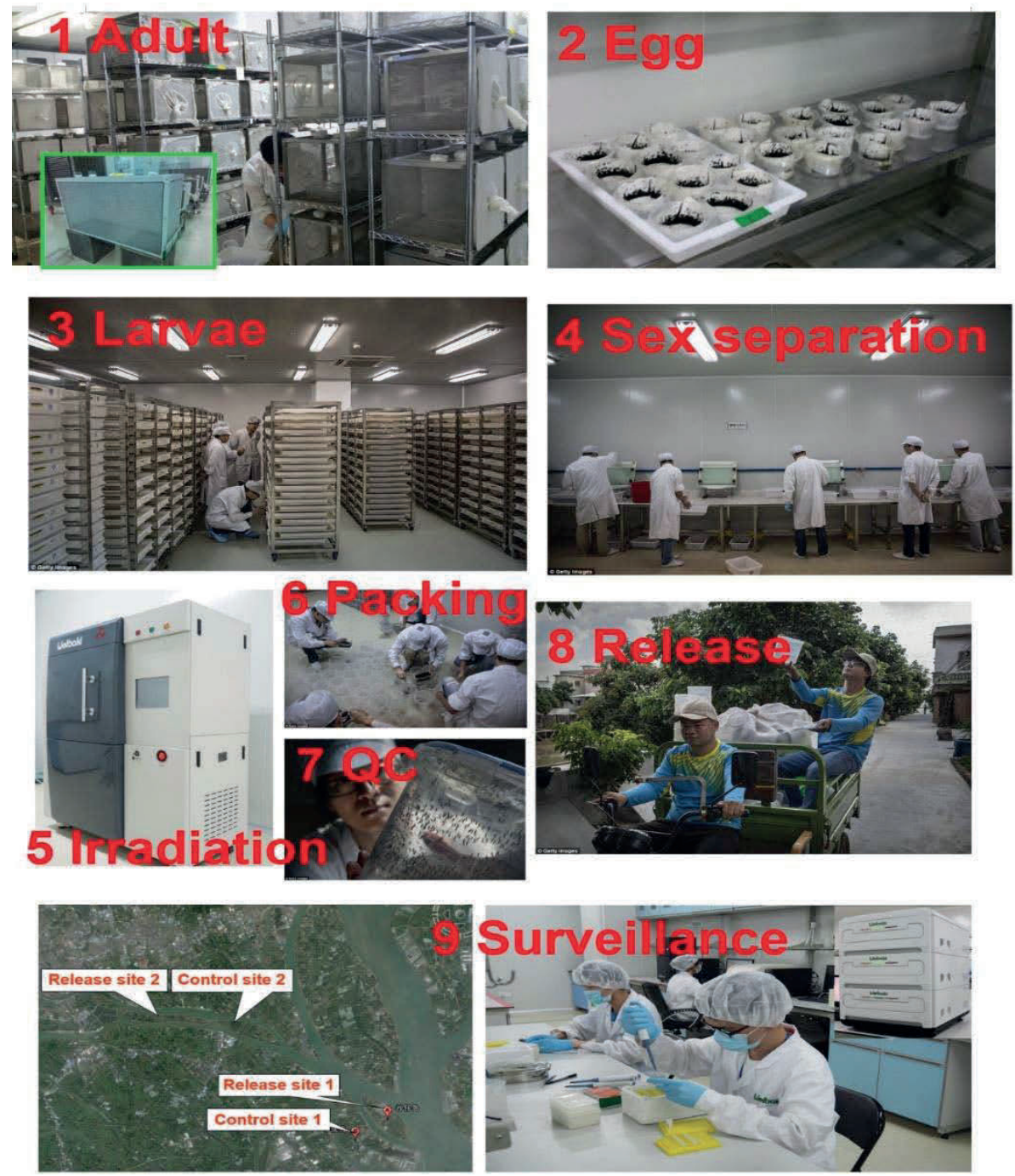

Figure 3. The different stages of the first combined IIT/SIT field trial against Ae. albopictus in Guangzhou, China. Photographs illustrating the nine different stages of the combined IIT/SIT field trial. In stage 1-3, artificially-triply-Wolbachia-infected adults, eggs and larvae were mass-produced in the mosquito rearing factory. In stage 4, a Fay-Morlan sorter was used for sex separation of pupae, followed by stage 5 with the Wolbaki ${ }^{\circledR} X$-ray irradiator custommade for the field trial to enable pupal irradiation. In stage 6, the sex-separated males were packed into buckets for mass-release. After quality control of emerged adult males by manual checking for contaminant adult females (stage 7), those buckets were delivered by vehicle to release sites (stage 8), as shown in the satellite images of the control and release sites (map data: Google, DigitalGlobe). Field populations were monitored through samples collected each week for diagnosis of $\mathrm{wPip}$ infection using PCR (stage 9). 
The "FAO/IAEA-unit" (Balestrino et al. 2012) holds 50 larval rearing trays, covers $0.94 \mathrm{~m}^{2}$ ground area, has a capacity to hold 900000 larvae, and can generate 314000 male pupae per rearing cycle (Zhang et al. 2017). The second generation "Wol-unit 2.0" is based on the FAO/IAEA-unit and holds 100 larval rearing trays, covers $1.2 \mathrm{~m}^{2}$ ground area, has a capacity to hold up to 1.5 million larvae, and can generate 550000 male pupae per rearing cycle. A comparison between these three larval rearing units for Ae. albopictus is summarized in Table 1. The Wol-unit 2.0 is recommended for medium to large-scale applications as it requires relatively less space and enables more male pupae to be generated per unit.

Table 1. Comparison between three larval rearing units for production of one million Aedes albopictus males

\begin{tabular}{|c|c|c|c|}
\hline Parameter & Wol-unit & FAO/IAEA-unit & Wol-unit 2.0 \\
\hline Number of trays per unit & 40 & 50 & 100 \\
\hline $\begin{array}{l}\text { Number of larvae reared } \\
\text { per unit }\left(10^{5}\right)\end{array}$ & 2.64 & 9.0 & 15.0 \\
\hline $\begin{array}{l}\text { Number of male pupae } \\
\text { acquired per unit }\left(10^{5}\right)\end{array}$ & 0.89 & 3.14 & 5.5 \\
\hline $\begin{array}{l}\text { Dimensions per unit } \\
(\mathrm{m}, \mathrm{L} * \mathrm{~W} * \mathrm{H})\end{array}$ & $0.97 \times 0.70 \times 1.85$ & $0.78 \times 1.2 \times 2.10$ & $1.41 \times 0.84 \times 2.1$ \\
\hline Ground area per unit $\left(\mathrm{m}^{2}\right)$ & 0.68 & 0.94 & 1.2 \\
\hline Quantity (unit) & 11.2 & 3.2 & 1.9 \\
\hline Total space $\left(\mathrm{m}^{2}\right)$ & 7.6 & 3.0 & 2.3 \\
\hline Labour - Adding water & Manual operation & $\begin{array}{l}\text { Semi-automatic } \\
\text { operation }\end{array}$ & $\begin{array}{l}\text { Semi-automatic } \\
\text { operation }\end{array}$ \\
\hline $\begin{array}{l}\text { Labour - Pupae/Larvae } \\
\text { collection }\end{array}$ & Manual operation & $\begin{array}{l}\text { Semi-automatic } \\
\text { operation }\end{array}$ & $\begin{array}{l}\text { Semi-automatic } \\
\text { operation }\end{array}$ \\
\hline Labour - Cleaning & Manual operation & $\begin{array}{l}\text { Semi-automatic } \\
\text { operation }\end{array}$ & $\begin{array}{l}\text { Semi-automatic } \\
\text { operation }\end{array}$ \\
\hline Price & Low & High & Medium \\
\hline Application & Small size factory & Medium size factory & $\begin{array}{l}\text { Medium/Large size } \\
\text { factory }\end{array}$ \\
\hline
\end{tabular}

For holding of adult mosquitoes, a suitable cage structure is important to maximize egg production. A prototype mass-production cage based on a design originally used for Mediterranean fruit flies, had been previously developed at the IPCL that allowed sugar and blood-feeding, as well as a simplified egg collection system that minimized the risk of mosquito escapes (Balestrino et al. 2014b; Mamai et al. 2017). However, we found that the egg production of Ae. albopictus was quite low, i.e. an average of $\sim 16$ eggs per female per blood meal. As rearing density seems to be the main factor causing low egg production (Balestrino et al. 2014b), the cage height was reduced and, together with the addition of ATP to the blood meal, we were able to increase 
egg production to an average of $\sim 70$ eggs per female given two blood meals (Zhang et al. 2018). The modified mass-production cage and mass-rearing protocol described currently enables the Wolbaki factory to produce 10 million Ae. albopictus eggs every 15 days (Zhang et al. 2018).

Male and female pupae of mosquitoes in the genera Aedes and Culex can be separated on the basis of size differences by using sieves or glass separators, although the traditional equipment is laborious to use (McCray 1961; Focks 1980; Balestrino et al. 2014a). An automated glass separator has been developed at Wolbaki to reduce manual operation and improve sex separation efficiency.

\subsection{Irradiation of Pupae for Release}

An irradiator specific for mosquito pupae was required for our field trial. Gamma rays have been the most common type of radiation used for insect sterilisation, because of their high energy and penetration (Bakri et al. 2021). However, the use of gamma rays is challenging because of regulatory, logistical and economic issues, related to safety, security, recycling, transportation, storage and initial cost. Consequently, in the past decade the use of X-rays has been suggested as a potential alternative to gamma rays (Mastrangelo et al. 2010; Ndo et al. 2014; Yamada et al. 2014a; FAO/IAEA 2017).

For insect sterilisation, a dose uniformity ratio (DUR: the maximum dose divided by the minimum dose) below 1.2 is required, in order to ensure a uniform dose is given to the irradiated individuals (Yamada et al. 2019). Dose uniformity is required to ensure that males do not receive unnecessarily high doses of radiation, which might needlessly reduce their quality, and is important for the combined IIT/SIT, where it is vital that all contaminant females are sufficiently irradiated to ensure complete sterilisation. However, the X-ray irradiators currently available on the market with the recommended DUR are not suitable for larger-scale applications using mosquitoes, because either only a small number of pupae can be simultaneously irradiated (RS 2000, Biological System Irradiator, RadSource, Georgia, USA), or they require relatively frequent replacement of the costly X-ray tube and are inconvenient for pupal irradiation (RS 2400) (Yamada et al. 2014a).

Consequently, Wolbaki in cooperation with the FAO/IAEA, developed a new Xray irradiator - "the Wolbaki irradiator" - specifically designed for pupal irradiation, which meets the technical requirements and large-scale processing capacity required for our field trial. The irradiator is equipped with a ray tube at a 40 -degree angle, and with a maximum power of $4.5 \mathrm{~kW}$. At a horizontal distance of $30 \mathrm{~cm}$ from the radiation source, the dose rate is measured at $3.2 \mathrm{~Gy} / \mathrm{min}$ through a $0.3 \mathrm{~mm}$ copper filter. A rotary table for holding canisters is set up for horizontal rotation during exposure. Two separated canisters, with a total loading capacity of one litre male pupae, can be vertically swapped at half target dose. The DUR is reduced to 1.07 by rotating and swapping during exposure.

As described above, the optimum irradiation dose for sterilizing of contaminant females for field release is likely to be appreciably higher than that indicated by a naïve interpretation of laboratory data based on very small sample sizes. Accordingly, we erred on the side of caution, and chose an irradiation dose of 45 Gy to ensure the success of our field trial (i.e. no fertile contaminant females released). 


\subsection{Open-Release in the Field and Entomological Surveillance}

The open-release field trial was undertaken over a 2 to 3 -year period (2016-2018) on two residential islands in Guangzhou, with each release site having its own control sites (Fig. 3) (Zheng et al. 2019). The field trial started a year earlier in Release Site 1 (2014 compared to 2015 for Release Site 2), with an initial pilot test of the IIT only in Release Site 1 during 2015, and a test of the combined IIT/SIT strategy being performed simultaneously in both sites during 2016 and 2017.

In the years prior to the male HC releases, base-line entomological surveys were carried out in both sets of control and release sites to confirm their suitability for the field trial (2014 for Site 1 and 2015 for Site 2; Fig. 4).

For the pilot test of IIT only, non-irradiated HC mosquitoes, from which the females had been removed by a combination of mechanical and manual sex sorting, were released during the mosquito breeding season (March to October). Initially, males were released throughout the entire area of Release Site 1, and the target field population was suppressed by as much as 55\% (March to May; Fig. 5). However, as the mosquito breeding season peaked (late May to early June), the level of population suppression diminished, as it was not possible to release sufficient numbers of Wolbachia-infected males throughout the entire release site in order to attain the critical overflooding ratio. This was due to the labour-intensive checks required to manually remove contaminant females from the released males, a rate-limiting step which constrained how many sex-sorted mosquitoes could be produced per week (given the number of staff available for our field trial). Therefore, in an attempt to achieve the critical overflooding ratio for the remainder of the IIT trial in 2015 (midJune to October), we reduced the treated area within Release Site 1 in which males were released (Fig. 4B), and subsequently expanded it following the "rolling carpet" approach (Dyck et al. 2021), so that the local density of released males would be increased, without the need to release a larger number of males overall.

After reducing the size of the release area, population suppression within the area of continuing releases was striking and significant, whereas very high mosquito densities were found in the immediately neighbouring area of Release Site 1 without continued releases (Fig. 4B), as well as within the control site (Fig. 5). These observations demonstrate the feasibility of using the IIT only for mosquito population suppression, and its potential for population elimination, if technological developments can be made that enable the large-scale mass-production - at a reasonable cost-effectiveness - of sufficient numbers of incompatible males lacking appreciable female contamination.

A trial of the combined IIT/SIT approach was then subsequently undertaken, in which irradiated $\mathrm{HC}$ mosquitoes were released. In this instance, females were removed from the released mosquitoes using mechanical separation only, resulting in a higher level of female contamination, but which could be tolerated as the residual females were sterilized by irradiation. As manual checks for contamination were no longer used or required, it was possible to release much larger numbers of male mosquitoes ( $>10$-fold) for the combined IIT/SIT approach than for the IIT alone, and so $\mathrm{HC}$ releases could be undertaken throughout the entire area of both release sites for the entire duration of the two-year combined IIT/SIT field trial. 
A

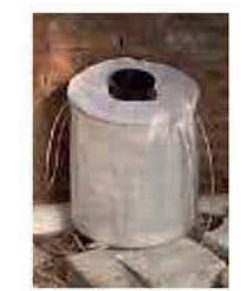

40 adult traps
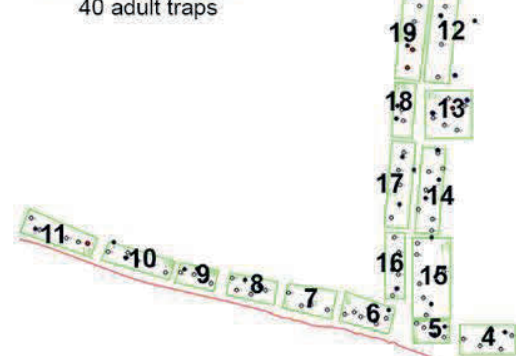

t:: $: 2 \cdot: 3 \cdots$

B

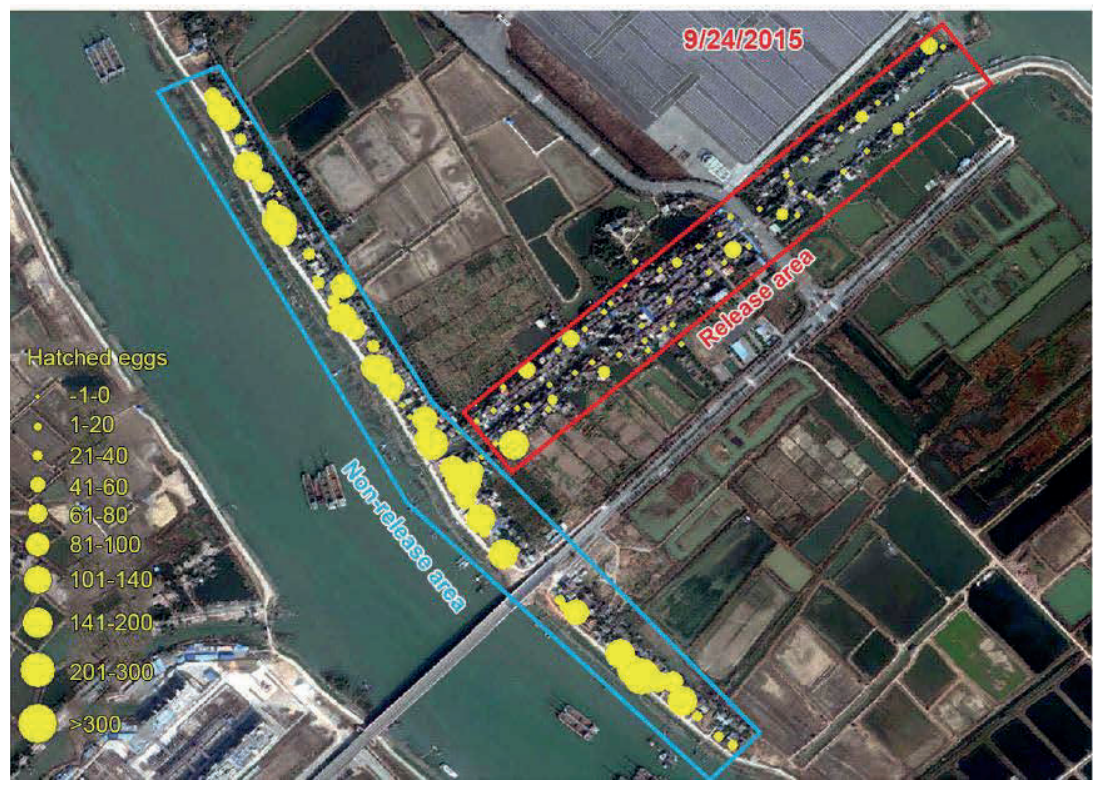

Figure 4. Monitoring and comparison of egg hatch rates during IIT and combined IIT/SIT application against Ae. albopictus in Release Site 1 in Guangzhou, China. (A) Schematic diagram illustrating the division of Release Site 1 into 22 zones (green boxes), and the location of the ovitraps and adult-collecting Biogents BG-Sentinel traps that were used weekly to monitor Aedes albopictus populations during the field trial. (B) Satellite image showing the non-release (blue box; zones 1 to 11) and release (red box; zones 12 to 22) areas within Release Site 1 during the IIT only phase of the field trial in September 2015 (Map data: Google, DigitalGlobe). Yellow circles indicate ovitrap locations, with areas proportional to the number of hatched eggs collected in each for that week in September 2015. 

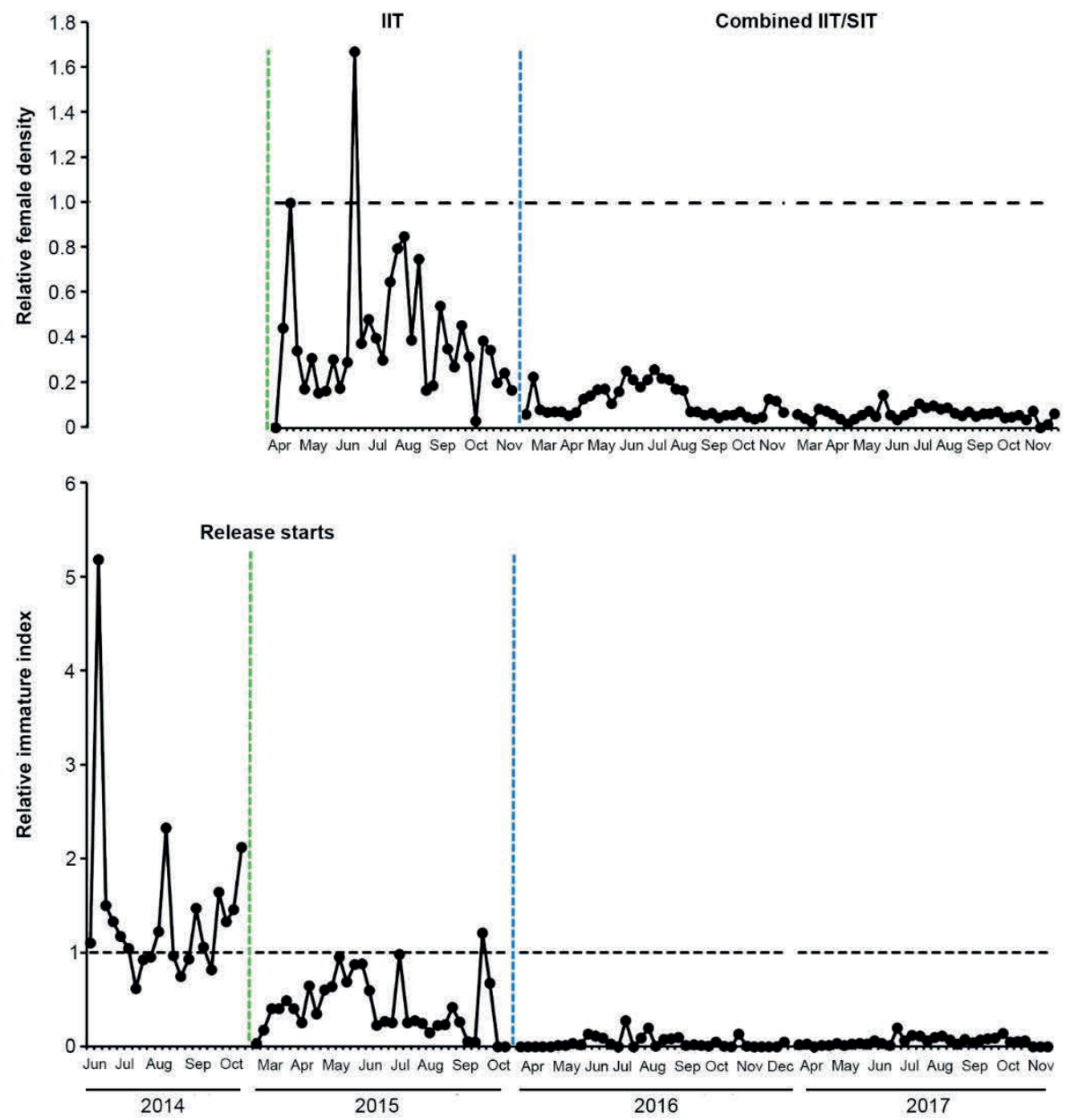

Figure 5. Suppression of Aedes albopictus in Release Site 1 during the field trial in Guangzhou, China. The solid black lines indicate, respectively, the densities of wild-type adult females $(A)$ and larvae $(B)$ collected in Release Site 1 standardized by dividing by the corresponding number of individuals collected in Control Site 1 (see stage 9 in Figure 3). In 2014, baseline data were collected during the "pre-release" period before any compatible males were released. In 2015, IIT only releases were undertaken. In 2016 and 2017, combined IIT/SIT releases were performed. The horizontal black dashed lines indicate the relative level of larvaeladults in Control Site 1. The vertical green dashed line indicates the onset of IIT-only releases of incompatible males, while the vertical blue dashed line indicates onset of combined IIT/SIT releases of irradiated incompatible males. 
Overall, during the mosquito breeding seasons of 2016 and 2017, over 197 million factory-reared irradiated $\mathrm{HC}$ males were released using buckets from which adults emerged (Fig. 3). On average, $0.2-0.3 \%$ of the released insects were contaminant females. The sterile to wild male overflooding ratio was estimated at between $8.7: 1$ to 15.8:1, which resulted in the near-elimination of wild-type adult female Ae. albopictus from both release sites, i.e. $>94 \%$ reduction in egg hatch and up to $94 \%$ reduction in the apparent density of wild adult females (Fig. 5). The failure to eliminate completely the target populations in our release sites appeared to have been due to a low level of immigration.

Importantly, we found no evidence of population replacement during the threeyear period of our field trial: throughout the period of male releases, we carefully monitored not only the wild-type target population, but also used PCR to screen collected larvae for the wPip Wolbachia strain infecting released HC individuals. Although we did find a very low level of $w$ Pip-positive larvae $(0.87 \%, 16 / 1844$ ovitrap samples), confirming the potential risk of population replacement, their collection was spatially and/or temporally-isolated, and they did not seem to constitute a viable breeding population. The field population did not increase in size after its initial suppression, nor was there a delayed rebound increase in egg hatch over time, which would have been expected, as a result of compatible matings becoming more frequent, if $w$ Pip-infected mosquitoes had established in the field.

Overall, these observations demonstrate that the combined IIT/SIT approach can (i) suppress and effectively eliminate mosquito vector populations, and (ii) provide protection against the risk of population replacement resulting from the accidental release of fertile compatible endosymbiont-infected females.

\section{FUTURE AREA-WIDE COMBINED IIT/IIT RELEASES}

Despite our successful field trial, doubts about the area-wide implementation of the combined IIT/SIT persist (Armbruster 2019). As with other sterile-male-based methods, concerns include the affordability and sustainability of large-scale massrelease programmes. We believe that with optimization of the protocols used in our field trial, the combined IIT/SIT approach can be both affordable and sustainable for lower-income countries, and have an important and leading role as part of area-wide integrated pest control programmes (see Supplementary Information to Zheng et al. 2019).

To completely remove the risk of population replacement and to obtain population suppression or elimination, pupae were irradiated with a relatively high dose in the mass-rearing facility (Zheng et al. 2019). In addition, the current design of the X-ray irradiator and canister require a large number (up to 200000 ) of pupae to stay in an overcrowded condition for an extended period (up to $15 \mathrm{~min}$ ). Both result in a negative impact on male mating competitiveness and reduced cost-effectiveness of combined IIT/SIT releases. Thus, efforts will be made to optimize the approach for radiation exposure and to further improve the design of the X-ray irradiator.

As with any sterile-male-based method, large-scale area-wide deployment of the combined IIT/SIT approach would benefit from the development of improved and/or new methods/technologies to facilitate the efficient mass-production and mass-release 
of sufficient incompatible males to achieve population suppression/elimination. Many of these requirements are not unique to combined IIT/SIT releases (Alphey et al. 2010; Bourtzis et al. 2016), and, as such, we do not review them here in detail, other than to indicate how improvements in sex separation might impact the combined IIT/SIT approach.

Although the development of perfect sex separation methods is highly desirable (Gilles et al. 2014), the existence of such methods would negate the need and necessity for combined IIT/SIT, enabling IIT to be conducted without the risk of accidental female release resulting in unintended population replacement, and enabling the SIT to be conducted without risk of increased pest/vector activity. However, as described by Franz et al. (2021), even the best genetic sexing systems available are not perfect under large-scale operational programmes. In addition, where CI-inducing endosymbionts and irradiation reduce the ability of insects to act as pests/vectors (Sections 3.4.2), the low levels of contaminant females is less problematic.

Improvements in sex separation are likely to have their greatest impact on combined IIT/SIT releases by enabling the application of this method to target species for which there are currently either insufficient sex separation methods to enable mass-releases, or they are not available (e.g. Anopheles mosquitoes, which cannot be easily separated on the basis of size).

\section{CONCLUSIONS}

The combined IIT/SIT strategy integrates the strengths of the IIT with those of the SIT, and in so doing overcomes the current technological limitations of each approach. It can be used as an environment-friendly biopesticide to meet the current need for a novel solution to suppress mosquito populations and their transmitted diseases. Our successful field trial demonstrates the feasibility of area-wide application of combined IIT/SIT releases for Aedes mosquitoes.

\section{REFERENCES}

Alam, U., J. Medlock, C. Brelsfoard, R. Pais, C. Lohs, S. Balmand, J. Carnogursky, A. Heddi, P. Takac, A. Galvani, and S. Aksoy. 2011. Wolbachia symbiont infections induce strong cytoplasmic incompatibility in the tsetse fly Glossina morsitans. PLoS Pathogens 7: e1002415.

Aliota, M. T., S. A. Peinado, I. D. Velez, and J. E. Osorio. 2016. The $w \mathrm{Mel}$ strain of Wolbachia reduces transmission of Zika virus by Aedes aegypti. Scientific Reports 6: 28792.

Alphey, L. 2014. Genetic control of mosquitoes. Annual Review of Entomology 59: 205-224.

Alphey, L., M. Benedict, R. Bellini, G. G. Clark, D. A. Dame, M. W. Service, and S. L. Dobson 2010. Sterile-insect methods for control of mosquito-borne diseases: An analysis. Vector-Borne and Zoonotic Diseases 10: 295-311.

Andreasen, M. H., and C. F. Curtis. 2005. Optimal life stage for radiation sterilisation of Anopheles males and their fitness for release. Medical and Veterinary Entomology 19: 238-244.

Andrews, E. S., P. R. Crain, Y. Fu, D. K. Howe, and S. L. Dobson. 2012. Reactive oxygen species production and Brugia pahangi survivorship in Aedes polynesiensis with artificial Wolbachia infection types. PLoS Pathogens 8: e1003075.

Anonymous. 1975. Oh New Delhi, Oh Geneva. Nature 256: 355-357.

Ant, T. H., and S. P. Sinkins. 2018. A Wolbachia triple-strain infection generates self-incompatibility in Aedes albopictus and transmission instability in Aedes aegypti. Parasites \& Vectors 11: 295. 
Apostolaki, A., I. Livadaras, A. Saridaki, A. Chrysargyris, C. Savakis, and K. Bourtzis. 2011. Transinfection of the olive fruit fly Bactrocera oleae with Wolbachia: Towards a symbiont-based population control strategy. Journal of Applied Entomology 135: 546-553.

Armbruster, P. A. 2019. Tiger mosquitoes tackled in a trial. Nature 572 (7767): 39-40.

Arunachalam, N., and C. F. Curtis. 1985. Integration of radiation with cytoplasmic incompatibility for genetic control in the Culex pipiens complex (Diptera: Culicidae). Journal of Medical Entomology 22: 648-653.

Atyame, C. M., N. Pasteur, E. Dumas, P. Tortosa, M. L. Tantely, N. Pocquet, S. Licciardi, A. Bheecarry, B. Zumbo, M. Weill, and O. Duron. 2011. Cytoplasmic incompatibility as a means of controlling Culex pipiens quinquefasciatus mosquito in the islands of the south-western Indian Ocean. PLoS Neglected Tropical Diseases 5: e1440.

Atyame, C. M., J. Cattel, C. Lebon, O. Flores, J. S. Dehecq, M. Weill, L. C. Gouagna, and P. Tortosa. 2015. Wolbachia-based population control strategy targeting Culex quinquefasciatus mosquitoes proves efficient under semi-field conditions. PLoS One 10: e0119288.

Atyame, C. M., P. Labbé, C. Lebon, M. Weill, R. Moretti, F. Marini, L. C. Gouagna, M. Calvitti, and P. Tortosa. 2016. Comparison of irradiation and Wolbachia based approaches for sterile-male strategies targeting Aedes albopictus. PLoS One 11: e0146834.

Axford, J. K., P. A. Ross, H. L. Yeap, A. G. Callahan, and A. A. Hoffmann. 2016. Fitness of $w$ AlbB Wolbachia infection in Aedes aegypti: Parameter estimates in an outcrossed background and potential for population invasion. The American Journal of Tropical Medicine and Hygiene 94: 507-516.

Bakri, A., N. Heather, J. Hendrichs, and I. Ferris. 2005. Fifty years of radiation biology in entomology: Lessons learned from IDIDAS. Annals of the Entomological Society of America 98: 1-12.

Bakri, A., K. Mehta, and D. R. Lance. 2021. Sterilizing insects with ionizing radiation, pp. 355-398. In V. A. Dyck, J. Hendrichs, and A. S. Robinson (eds.), Sterile Insect Technique - Principles and practice in Area-Wide Integrated Pest Management. Second Edition. CRC Press, Boca Raton, Florida, USA.

Balestrino, F., M. Q. Benedict, and J. R. L. Gilles. 2012. A new larval tray and rack system for improved mosquito mass rearing. Journal of Medical Entomology 49: 595-605.

Balestrino, F., A. Puggioli, J. R. L. Gilles, and R. Bellini. 2014a. Validation of a new larval rearing unit for Aedes albopictus (Diptera: Culicidae) mass rearing. PLoS One 9: e91914.

Balestrino, F., I. A. Puggioli, R. Bellini, D. Petric, and J. R. L. Gilles. 2014b. Mass production cage for Aedes albopictus (Diptera: Culicidae). Journal of Medical Entomology 51: 155-163.

Balestrino, F., A. Medici, G. Candini, M. Carrieri, B. Maccagnani, M. Calvitti, S. Maini, and R. Bellini. 2010. Gamma ray dosimetry and mating capacity studies in the laboratory on Aedes albopictus males. Journal of Medical Entomology 47: 581-591.

Barclay, H. J. 2021. Mathematical models for using sterile insects, pp. 201-244. In V. A. Dyck, J. Hendrichs, and A. S. Robinson (eds.), Sterile Insect Technique - Principles and practice in Area-Wide Integrated Pest Management. Second Edition. CRC Press, Boca Raton, Florida, USA.

Barr, A. R. 1966. Cytoplasmic incompatibility as a means of eradication of Culex pipiens L. Proceedings and Papers of the Annual Conference of the California Mosquito Control Association 34: 32-35.

Barr, A. R. 1970. Partial compatibility and its effect on eradication by the incompatible male method. Proceedings and Papers of the Annual Conference of the California Mosquito Control Association (Thirty-Seventh Annual Conference, Los Angeles, 27-29th January 1969) 37: 19-24.

Baton, L.A., E. C. Pacidônio, D. da Silva Gonçalves, and L. A. Moreira. 2013. wFlu: Characterization and evaluation of a native Wolbachia from the mosquito Aedes fluviatilis as a potential vector control agent. PLoS One 8: e59619.

Bellini, R., M. Calvitti, A. Medici, M. Carrieri, G. Celli, and S. Maini. 2007. Use of the sterile insect technique against Aedes albopictus in Italy: First results of a pilot trial, pp. 505-515. In M. J. B. Vreysen, A. S. Robinson, and J. Hendrichs (eds.), Area-wide control of insect pests: From research to field implementation. Springer, Dordrecht, The Netherlands.

Bellini, R., F. Balestrino, A. Medici, G. Gentile, R. Veronesi, and M. Carrieri. 2013a. Mating competitiveness of Aedes albopictus radio-sterilized males in large enclosures exposed to natural conditions. Journal of Medical Entomology 50: 94-102.

Bellini, R., A. Medici, A. Puggioli, F. Balestrino, and M. Carrieri. 2013b. Pilot field trials with Aedes albopictus irradiated sterile males in Italian urban areas. Journal of Medical Entomology 50: 317-325.

Benedict, M. Q., and A. S. Robinson. 2003. The first releases of transgenic mosquitoes: An argument for the sterile insect technique. Trends in Parasitology 19: 349-355. 
Berryman, A. A., T. P. Boo'co, and L. C. Dickmann. 1973. Computer simulation of population reduction by release of sterile insects: II. The effects of dynamic survival and multiple mating, pp. 31-43. In Computer models and application of the Sterile-Male Technique: Proceedings of a Panel, Vienna, $13-$ 17 December 1971, organized by the Joint FAO/IAEA Division of Atomic Energy in Food and Agriculture, International Atomic Energy Agency, Vienna, Austria.

Bian, G., Y. Xu, P. Lu, Y. Xie, and Z. Xi. 2010. The endosymbiotic bacterium Wolbachia induces resistance to dengue virus in Aedes aegypti. PLoS Pathogens 6: e1000833.

Bian, G., D. Joshi, Y. Dong, P. Lu, G. Zhou, X. Pan, Y. Xu, G. Dimopoulos, and Z. Xi. 2013. Wolbachia invades Anopheles stephensi populations and induces refractoriness to Plasmodium infection. Science 340: (6133) 748-751.

Blagrove, M. S. C., C. Arias-Goeta, A.-B. Failloux, and S. P. Sinkins. 2012. Wolbachia strain wMel induces cytoplasmic incompatibility and blocks dengue transmission in Aedes albopictus. Proceedings of the National Academy of Sciences of the USA 109: 255-260.

Blagrove, M. S. C., C. Arias-Goeta, C. Di Genua, A.-B. Failloux, and S. P. Sinkins. 2013. A Wolbachia $w \mathrm{Mel}$ transinfection in Aedes albopictus is not detrimental to host fitness and inhibits Chikungunya virus. PLoS Neglected Tropical Diseases 7: e2152.

Blümel, S., and K. Russ. 1989. Manipulation of races, pp. 387-389. In A. S. Robinson and G. Hooper (eds.), Fruit flies: Their biology, natural enemies and control. World Crop Pests, Volume 3B. Elsevier, Amsterdam, The Netherlands.

Boller, E. F. 1989. Cytoplasmic incompatibility in Rhagoletis cerasi, pp. 69-74. In A. S. Robinson and G. Hooper (eds.), Fruit flies: Their biology, natural enemies and control. World Crop Pests, Volume 3B. Elsevier, Amsterdam, The Netherlands.

Boller, E. F., and G. L. Bush. 1974. Evidence for genetic variation in populations of the European cherry fruit fly, Rhagoletis cerasi (Diptera: Tephritidae) based on physiological parameters and hybridization experiments. Entomologia Experimentalis et Applicata 17: 279-293.

Boller, E. F., K. Russs, V. Vallo, and G. L. Bush. 1976. Incompatible races of European cherry fruit fly, Rhagoletis cerasi (Diptera: Tephritidae), their origin and potential use in biological control. Entomologia Experimentalis et Applicata 20: 237-247.

Bourtzis, K. and A. S. Robinson. 2006. Insect pest control using Wolbachia and/or radiation, pp. 225246. In K. Bourtzis and T. A. Miller (eds.), Insect symbiosis. Volume 2. CRC Press, Boca Raton, USA.

Bourtzis, K., R. S. Lees, J. Hendrichs, and M. J. B. Vreysen. 2016. More than one rabbit out of the hat: Radiation, transgenic and symbiont-based approaches for sustainable management of mosquito and tsetse fly populations. Acta Tropica 157: 115-130.

Bourtzis, K., S. L. Dobson, Z. Xi, J. L. Rasgon, M. Calvitti, L. A. Moreira, H. Bossin, R. Moretti, L. A. Baton, G. L. Hughes, P. Mavingui, and J. R. L. Gilles. 2014. Harnessing mosquito-Wolbachia symbiosis for vector and disease control. Acta Tropica 132 (Supplement): S150-S163.

Boyle, L., S. L. O'Neill, H. M. Robertson, and T. L. Karr. 1993. Interspecific and intraspecific horizontal transfer of Wolbachia in Drosophila. Science 260: (5115) 1796-1799.

Braig, H. R., H. Guzman, R. B. Tesh, and S. L. O'Neill. 1994. Replacement of the natural Wolbachia symbiont of Drosophila simulans with a mosquito counterpart. Nature 367: (6462) 453-455.

Brelsfoard, C. L., Y. Séchan, and S. L. Dobson. 2008. Interspecific hybridization yields strategy for South Pacific filariasis vector elimination. PLoS Neglected Tropical Diseases 2: e129.

Brelsfoard, C. L., W. St Clair, and S. L. Dobson. 2009. Integration of irradiation with cytoplasmic incompatibility to facilitate a lymphatic filariasis vector elimination approach. Parasites \& Vectors 2 : (1) 38

Brooks, G. D., C. F. Curtis, K. K. Grover, B. S. Krishnamurthy, P. L. Rajagopalan, L. S. Sharma, V. P. Sharma, D. Singh, K. R. P. Singh, M. Yasuno, M. A. Ansari, T. Adak, H. V. Aggarwal, C. P. Batra, R. K. Chandrahas, P. R. Malhotra, P. K. B. Menon, R. Menon, S. Das, R. K. Razdan, and V. Vaidyanathan. 1976. A field trial on control of Culex pipens fatigans Wied. by release of males of a strain integrating cytoplasmic incompatibility and a translocation. WHO/VBC/76.635. World Health Organization, Geneva, Switzerland.

Brower, J. H. 1976. Cytoplasmic incompatibility: Occurrence in a stored product pest Ephestia cautella. Annals of the Entomological Society of America 69: 1011-1015.

Brower, J. H. 1978. Propensity of interstrain mating in cytoplasmically incompatible strains of the almond moth. Journal of Economic Entomology 71: 585-586.

Brower, J. H. 1979. Suppression of laboratory populations of Ephestia cautella (Walker) (Lepidoptera: Pyralidae) by release of males with cytoplasmic incompatibility. J. Stored Products Research 15: 1-4. 
Brower, J. H. 1980. Reduction of almond moth populations in simulated storages by the release of genetically incompatible males. Journal of Economic Entomology 73: 415-418.

Brownstein, J. S., E. Hett, and S. L. O'Neill. 2003. The potential of virulent Wolbachia to modulate disease transmission by insects. Journal of Invertebrate Pathology 84: 24-29.

Calvitti, M., R. Moretti, D. Porretta, R. Bellini, and S. Urbanelli. 2009. Effects on male fitness of removing Wolbachia infections from the mosquito Aedes albopictus. Medical and Veterinary Entomology 23: 132-140.

Calvitti, M., R. Moretti, E. Lampazzi, R. Bellini, and S. L. Dobson. 2010. Characterization of a new Aedes albopictus (Diptera: Culicidae)-Wolbachia pipientis (Rickettsiales: Rickettsiaceae) symbiotic association generated by artificial transfer of the wPip strain from Culex pipiens (Diptera: Culicidae). Journal of Medical Entomology 47: 179-187.

Calvitti, M., R. Moretti, A. R. Skidmore, and S. L. Dobson. 2012. Wolbachia strain $w$ Pip yields a pattern of cytoplasmic incompatibility enhancing a Wolbachia-based suppression strategy against the disease vector Aedes albopictus. Parasites \& Vectors 5: 254.

Calvitti, M., F. Marini, A. Desiderio, A. Puggioli, and R. Moretti. 2015. Wolbachia density and cytoplasmic incompatibility in Aedes albopictus: Concerns with using artificial Wolbachia infection as a vector suppression tool. PLoS One 10: e 0121813.

Caspari, E., and G. S. Watson. 1959. On the evolutionary importance of cytoplasmic sterility in mosquitoes. Evolution 13: 568-570.

Cattel, J., K. Nikolouli, T. Andrieux, J. Martinez, F. Jiggins, S. Charlat, F. Vavre, D. Lejon, P. Gibert, and L. Mouton. 2018. Back and forth Wolbachia transfers reveal efficient strains to control spotted wing drosophila populations. Journal of Applied Ecology 55: 2408-2418.

Chen, L., C. Zhu, and D. Zhang. 2013. Naturally occurring incompatibilities between different Culex pipiens pallens populations as the basis of potential mosquito control measures. PLoS Neglected Tropical Diseases 7: e2030.

Cook, P. E., C. J. McMeniman, and S. L. O'Neill. 2008. Modifying insect population age structure to control vector-borne disease. Advances in Experimental Medicine and Biology 627: 126-140.

Corbel, V., C. Durot, N. L. Achee, F. Chandre, M. B. Coulibaly, J. P. David, G. J. Devine, I. Dusfour, D. M. Fonseca, J. Griego, W. Juntarajumnong, A. Lenhart, S. Kasai, A. J. Martins, C. Moyes, L. C. Ng, J. Pinto, J. F. Pompon, P. Muller, K. Raghavendra, D. Roiz, H. Vatandoost, J. Vontas, and D. Weetman. 2019. Second WIN International Conference on "Integrated approaches and innovative tools for combating insecticide resistance in vectors of arboviruses". 1-3 October 2018, Singapore. Parasites \& Vectors 12 (1): 331.

Curtis, C. F. 1977. Testing systems for the genetic control of mosquitoes, pp. 106-116. In J. S. Packer and D. White (eds.), XV International Congress of Entomology, Entomological Society of America, College Park, Maryland, USA.

Curtis, C. F., and R. Reuben. 2007. Destruction in the 1970s of a research unit in India on genetic control of mosquitoes and a warning for the future management of transgenic research. Antenna 31:214-216.

Curtis, C. F., G. D. Brooks, M. A. Ansari, K. K. Grover, B. S. Krishnamurthy, P. K. Rajagopalan, L. S. Sharma, V. P. Sharma, D. Singh, K. R. P. Singh, and M. Yasuno. 1982. A field trial on control of Culex quinquefasciatus by release of males of a strain integrating cytoplasmic incompatibility and a translocation. Entomologia Experimentalis et Applicata 31: 181-190.

Curtis, C. F., D. S. Ellis, P. E. Doyle, N. Hill, B. D. Ramji, L. W. Irungu, and H. Townson. 1983. Susceptibility of aposymbiotic Culex quinquefasciatus to Wuchereria bancrofti. Journal of Invertebrate Pathology 41: 214-223.

Dame, D. A., C. F. Curtis, M. Q. Benedict, A. S. Robinson, and B. G. Knols. 2009. Historical applications of induced sterilisation in field populations of mosquitoes. Malaria Journal 8 (Supplement 2): S2.

Davidson, G. 1974. Genetic control of insect pests. Academic Press, London, UK. 168 pp.

Dobson, S. L., and W. Rattanadechakul. 2001. A novel technique for removing Wolbachia infections from Aedes albopictus (Diptera: Culicidae). Journal of Medical Entomology 38: 844-849.

Dobson, S. L., C. W. Fox, and F. M. Jiggins. 2002a. The effect of Wolbachia-induced cytoplasmic incompatibility on host population size in natural and manipulated systems. Proceedings of the Royal Society, Series B: Biological Sciences 269: (1490) 437-445.

Dobson, S. L., E. J. Marsland, and W. Rattanadechakul. 2002b. Mutualistic Wolbachia infection in Aedes albopictus: Accelerating cytoplasmic drive. Genetics 160: 1087-1094. 
Dutra, H. L. C., M. N. Rocha, F. B. S. Dias, S. B. Mansur, E. P. Caragata, and L. A. Moreira. 2016. Wolbachia blocks currently circulating Zika virus isolates in Brazilian Aedes aegypti mosquitoes. Cell Host and Microbe 19: 771-774.

Dutton, T. J., and S. P. Sinkins. 2005. Filarial susceptibility and effects of Wolbachia in Aedes pseudoscutellaris mosquitoes. Medical and Veterinary Entomology 19: 60-65.

Dyck, V. A., J. Hendrichs, and A. S. Robinson (eds.). 2021. Sterile Insect Technique - Principles and practice in Area-Wide Integrated Pest Management, Second Edition. CRC Press, Boca Raton, Florida, USA. $1200 \mathrm{pp}$.

(FAO/IAEA) Food and Agriculture Organization / International Atomic Energy Agency. 2017. Technical specification for an X-ray system for the irradiation of insects for the sterile insect technique and other related technologies. Joint FAO/IAEA Programme of Nuclear Techniques in Food and Agriculture, Vienna, Austria. 11 pp.

Fine, P. E. M. 1978. On the dynamics of symbiote-dependent cytoplasmic incompatibility in culicine mosquitoes. Journal of Invertebrate Pathology 31: 10-18.

Focks, D. A. 1980. An improved separator for the developmental stages, sexes, and species of mosquitoes (Diptera: Culicidae). Journal of Medical Entomology 17: 567-568.

Franz, F., K. Bourtzis, and C. Cáceres. 2021. Practical and operational genetic sexing systems based on classic genetic approaches in fruit flies, an example for other species amenable to large-scale rearing for the Sterile Insect Technique, pp. 575-604. In V. A. Dyck, J. Hendrichs, and A. S. Robinson (eds.), Sterile Insect Technique - Principles and practice in Area-Wide Integrated Pest Management. Second Edition. CRC Press, Boca Raton, Florida, USA.

Fu, Y., L. Gavotte, D. R. Mercer, and S. L. Dobson. 2010. Artificial triple Wolbachia infection in Aedes albopictus yields a new pattern of unidirectional cytoplasmic incompatibility. Applied and Environmental Microbiology 76: 5887-5891.

Gilles, J. R. L., M. F. Schetelig, F. Scolari, F. Marec, M. L. Capurro, G. Franz, and K. Bourtzis. 2014. Towards mosquito sterile insect technique programmes: Exploring genetic, molecular, mechanical and behavioural methods of sex separation in mosquitoes. Acta Tropica 132 (Supplement): S178-S187.

Glaser, R. L., and M. A. Meola. 2010. The native Wolbachia endosymbionts of Drosophila melanogaster and Culex quinquefasciatus increase host resistance to West Nile virus infection. PLoS One 5: e11977.

Graham, R. I., D. Grzywacz, W. L. Mushobozi, K. Wilson, and D. Ebert. 2012. Wolbachia in a major African crop pest increases susceptibility to viral disease rather than protects. Ecology Letters 15: 993 1000 .

Grover, K. K., and V. P. Sharma. 1974. The present status of the work on induced sterility mechanisms for control of mosquitoes Culex pipiens fatigans and Aedes aegypti at the WHO/ICMR Research Unit on Genetic Control of Mosquitoes. The Journal of Communicable Diseases 6: 91-97.

Grover, K. K., C. F. Curtis, V. P. Sharma, K. R. P. Singh, K. Dietz, H. V. Aggarwal, R. K. Razdan, and V. Vaidyanathan. 1976. Competitiveness of chemosterilised males and cytoplasmically incompatible translocated males of Culex pipiens fatigans Wiedemann (Diptera, Culicidae) in the field. Bulletin of Entomological Research 66: 469-480.

Hancock, P. A., S. P. Sinkins, and H. C. J. Godfray. 2011. Strategies for introducing Wolbachia to reduce transmission of mosquito-borne diseases. PLoS Neglected Tropical Diseases 5: e1024.

Hedges, L. M., J. C. Brownlie, S. L. O'Neill, and K. N. Johnson. 2008. Wolbachia and virus protection in insects. Science 322: (5902) 702.

Helinski, M. E. H., and B. G. J. Knols. 2008. Mating competitiveness of male Anopheles arabiensis mosquitoes irradiated with a partially or fully sterilizing dose in small and large laboratory cages. Journal of Medical Entomology 45: 698-705.

Helinski, M. E. H., A. G. Parker, and B. G. J. Knols. 2006. Radiation-induced sterility for pupal and adult stages of the malaria mosquito Anopheles arabiensis. Malaria Journal 5: 41.

Helinski, M. E. H., A. G. Parker, and B. G. J. Knols. 2009. Radiation biology of mosquitoes. Malaria Journal 8 (Supplement 2): S6.

Hertig, M., 1936. The rickettsia, Wolbachia pipientis (gen. et sp.n.) and associated inclusions of the mosquito, Culex pipiens. Parasitology 28: 453-486.

Hertig, M., and S. B. Wolbach. 1924. Studies on rickettsia-like micro-organisms in insects. The Journal of Medical Research 44: 329-374.

Hilgenboecker, K., P. Hammerstein, P. Schlattmann, A. Telschow, and J. H. Werren. 2008. How many species are infected with Wolbachia? A statistical analysis of current data. FEMS Microbiology Letters 281: 215-220. 
Hoffmann, A. A., P. A. Ross, and G. Rasic. 2015. Wolbachia strains for disease control: Ecological and evolutionary considerations. Evolutionary Applications 8: 751-768.

Hoffmann, A. A., B. L. Montgomery, J. Popovici, I. Iturbe-Ormaetxe, P. H. Johnson, F. Muzzi, M. Greenfield, M. Durkan, Y. S. Leong, Y. Dong, H. Cook, J. Axford, A. G. Callahan, N. Kenny, C. Omodei, E. A. McGraw, P. A. Ryan, S. A. Ritchie, M. Turelli, and S. L. O'Neill. 2011. Successful establishment of Wolbachia in Aedes populations to suppress dengue transmission. Nature 476: (7361) 454- 457.

Hoffmann, A. A., I. Iturbe-Ormaetxe, A. G. Callahan, B. L. Phillips, K. Billington, J. K. Axford, B. Montgomery, A. P. Turley, and S. L. O'Neill. 2014. Stability of the wMel Wolbachia infection following invasion into Aedes aegypti populations. PLoS Neglected Tropical Diseases 8: e3115.

Hughes, G. L., and J. L. Rasgon. 2014. Transinfection: A method to investigate Wolbachia-host interactions and control arthropod-borne disease. Insect Molecular Biology 23: 141-151.

Hurst, G. D. D., and C. L. Frost. 2015. Reproductive parasitism: Maternally inherited symbionts in a biparental world. Cold Spring Harbor Perspectives in Biology 7: a017699.

Iturbe-Ormaetxe, I., T. Walker, and S. L. O'Neill. 2011. Wolbachia and the biological control of mosquito-borne disease. EMBO Reports 12: 508-518.

Joshi, D., M. J. McFadden, D. Bevins, F. Zhang, and Z. Xi. 2014. Wolbachia strain wAlbB confers both fitness costs and benefit on Anopheles stephensi. Parasites \& Vectors 7: 336.

Joshi, D., X. Pan, M. J. McFadden, D. Bevins, X. Liang, P. Lu, S. Thiem, and Z. Xi. 2017. The maternally inheritable Wolbachia wAlbB induces refractoriness to Plasmodium berghei in Anopheles stephensi. Frontiers in Microbiology 8: 366

Joubert, D. A., T. Walker, L. B. Carrington, J. T. De Bruyne, D. H. Kien, N. T. Hoang, N. V. Chau, I. Iturbe-Ormaetxe, C. P. Simmons, and S. L. O'Neill. 2016. Establishment of a Wolbachia superinfection in Aedes aegypti mosquitoes as a potential approach for future resistance management. PLoS Pathogens 12: e1005434.

Juan-Blasco, M., A. Urbaneja, V. San Andrés, P. Castañera, and B. Sabater-Muñoz. 2013. Improving the sterile sperm identification method for its implementation in the area-wide sterile insect technique program against Ceratitis capitata (Diptera: Tephritidae) in Spain. Journal of Economic Entomology 106: 2541-2547.

Kariithi, H. M., I. K. Meki, D. I. Schneider, L. De Vooght, F. M. Khamis, A. Geiger, G. DemirbaşUzel, J. M. Vlak, I. A. Ince, S. Kelm, F. Njiokou, F. N. Wamwiri, I. I. Malele, B. L. Weiss, and A. M. M. Abd-Alla. 2018. Enhancing vector refractoriness to trypanosome infection: Achievements, challenges and perspectives. BMC Microbiology 18 (Supplement 1): 179.

Kambris, Z., P. E. Cook, H. K. Phuc, and S. P. Sinkins. 2009. Immune activation by life-shortening Wolbachia and reduced filarial competence in mosquitoes. Science 326: (5949) 134-136.

Kittayapong, P., N. Kaeothaisong, S. Ninphanomchai, and W. Limohpasmanee. 2018. Combined sterile insect technique and incompatible insect technique: Sex separation and quality of sterile Aedes aegypti male mosquitoes released in a pilot population suppression trial in Thailand. Parasites \& Vectors 11 (Supplement 2): 657.

Kittayapong, P., S. Ninphanomchai, W. Limohpasmanee, C. Chansang, U. Chansang, and P. Mongkalangoon. 2019. Combined sterile insect technique and incompatible insect technique: The first proof-of-concept to suppress Aedes aegypti vector populations in semi-rural settings in Thailand. PLoS Neglected Tropical Diseases 13: e0007771.

Knipling, E. F. 1955. Possibilities of insect control or eradication through the use of sexually sterile males. Journal of Economic Entomology 48: 459-462.

Knipling, E. F., H. Laven, G. B. Craig, R. Pal, J. B. Kitzmiller, C. N. Smith, and A. W. A. Brown. 1968. Genetic control of insects of public health importance. Bulletin of the World Health Organization 38: 421-438.

Krishnamurthy, B. S. 1977. Evaluation of the D3 cytoplasmic incompatible strain of Culex pipiens fatigans in laboratory and field cages. The Indian Journal of Medical Research 65 (Supplement): 1320.

Krishnamurthy, B. S., and H. Laven. 1976. Development of cytoplasmically incompatible and integrated (translocated incompatible) strains of Culex pipiens fatigans for use in genetic control. Journal of Genetics 62: 117-129.

Krishnamurthy, B. S., C. F. Curtis, S. K. Subbarao, K. R. P. Singh, R. K. Chandrahas, and T. Adak. 1977. Further studies on the effect of aging and mating history of males on cytoplasmic incompatibility in Culex pipiens fatigans. Journal of Genetics 63: 31-37. 
Laird, M. 1967. Eradication of Culex pipiens fatigans through cytoplasmic incompatibility. Nature 216 (5122) 1358.

Laven, H. 1967. Eradication of Culex pipiens fatigans through cytoplasmic incompatibility. Nature 216 : (5113) 383-384.

Laven, H. 1971. Une expérience de lutte génétique contre Culex pipiens fatigans Wied. 1828. Annales de Parasitologie Humaine et Comparée 46 (3, Supplément): 117-148.

Laven, H. 1974. Genetic control of mosquitoes, pp. 19-26. In Proceedings Tall Timbers Conference on Ecological Animal Control by Habitat Management, Number 5. Tall Timbers Research Station, Tallahassee, Florida, USA.

Laven, H., and M. Aslamkhan. 1970. Control of Culex pipiens pipiens and C. p. fatigans with integrated genetical systems. Pakistan Journal of Science 22: 303-312.

Lees, R. S., D. Carvalho, and J. Bouyer. 2021. Potential impact of the fight against disease-transmitting mosquitoes using the sterile insect technique, pp. 1081-1118. In V. A. Dyck, J. Hendrichs, and A. S. Robinson (eds.), Sterile Insect Technique - Principles and practice in Area-Wide Integrated Pest Management. Second Edition. CRC Press, Boca Raton, Florida, USA.

Lees R. S., B. Knols, R. Bellini, M. Q. Benedict, A. Bheecarry, H. C. Bossin, D. D. Chadee, J. Charlwood, R. K. Dabiré, L. Djogbenou, A. Egyir-Yawson, R. Gato, L. C. Gouagna, M. M. Hassan, S. A. Khan, L. L. Koekemoer, G. Lemperiere, N. C. Manoukis, and J. R. L. Gilles. 2013. Review: Improving our knowledge of male mosquito biology in relation to genetic control programmes. Acta Tropica 13 (Supplement): S2-S11.

Lees, R. S., J. R. L Gilles, J. Hendrichs, M. J. B. Vreysen, and K. Bourtzis. 2015. Back to the future: The Sterile Insect Technique against mosquito disease vectors. Current Opinion in Insect Science 10: $156-162$.

Maïga, H., D. Damiens, A. Niang, S. P. Sawadogo, O. Fatherhaman, R. S. Lees, O. Roux, R. K. Dabiré, G. A. Ouedraogo, F. Tripet, A. Diabate, and J. R. L. Gilles. 2014. Mating competitiveness of sterile male Anopheles coluzzii in large cages. Malaria Journal 13: 460.

Mains, J. W., C. L. Brelsfoard, R. I. Rose, and S. L. Dobson. 2016. Female adult Aedes albopictus suppression by Wolbachia-infected male mosquitoes. Scientific Reports 6: 33846.

Mains, J. W., P. H. Kelly, K. L. Dobson, W. D. Petrie, and S. L. Dobson. 2019. Localized control of Aedes aegypti (Diptera: Culicidae) in Miami, FL, via inundative releases of Wolbachia-infected male mosquitoes. Journal of Medical Entomology 56: 1296-1303.

Mamai, W., N. S. Bimbile-Somda, H. Maiga, J. G. Juarez, Z. A. I. Muosa, A. B. Ali, R. S. Lees, and J. R. L. Gilles. 2017. Optimization of mosquito egg production under mass rearing setting: Effects of cage volume, blood meal source and adult population density for the malaria vector, Anopheles arabiensis. Malaria Journal 16: 41.

Marshall, J. F. 1938. The British Mosquitoes. William Clowes and Sons, Limited, London and Beccles, UK. 341 pp.

Marshall, J. F., and J. Staley. 1937. Some notes regarding the morphological and biological differentiation of Culex pipiens Linnaeus and Culex molestus Forskål (Diptera, Culicidae). Proceedings of the Royal Entomological Society of London, Series A: General Entomology 12: 17-26.

Mastrangelo, T., A. G. Parker, A. Jessup, R. Pereira, D. Orozco-Davila, A. Islam, T. Dammalage, and $\mathbf{J}$. Walder. 2010. A new generation of $\mathrm{x}$ ray irradiators for insect sterilisation. Journal of Economic Entomology 103: 85-94.

McCray, E. M. 1961. A mechanical device for the rapid sexing of Aedes aegypti pupae. Journal of Economic Entomology 54: 819.

McMeniman, C. J., R. V. Lane, B. N. Cass, A. W. C. Fong, M. Sidhu, Y.-F. Wang, and S. L. O'Neill. 2009. Stable introduction of a life-shortening Wolbachia infection into the mosquito Aedes aegypti. Science 323: (5910) 141-144.

Min, K.-T., and S. Benzer. 1997. Wolbachia, normally a symbiont of Drosophila, can be virulent, causing degeneration and early death. Proceedings of the National Academy of Sciences of the USA 94: 1079210796.

Moreira, L.A., I. Iturbe-Ormaetxe, J. A. Jeffery, G. Lu, A. T. Pyke, L. M. Hedges, B. C. Rocha, S. Hall-Mendelin, A. Day, M. Riegler, L. E. Hugo, K. N. Johnson, B. H. Kay, A. E. McGraw, A. F. van den Hurk, P. A. Ryan, and S. L. O'Neill. 2009. A Wolbachia symbiont in Aedes aegypti limits infection with dengue, Chikungunya, and Plasmodium. Cell 139: 1268-1278.

Moretti, R., and M. Calvitti. 2013. Male mating performance and cytoplasmic incompatibility in a $w$ Pip Wolbachia trans-infected line of Aedes albopictus (Stegomyia albopicta). Medical and Veterinary Entomology 27: 377-386. 
Moretti, R., G. A. Marzo, E. Lampazzi, and M. Calvitti. 2018a. Cytoplasmic incompatibility management to support Incompatible Insect Technique against Aedes albopictus. Parasites \& Vectors 11 (Supplement 2): 649.

Moretti, R., P.-S. Yen, V. Houé, E. Lampazzi, A. Desiderio, A.-B. Failloux, and M. Calvitti. 2018 b. Combining Wolbachia-induced sterility and virus protection to fight Aedes albopictus-borne viruses. PLoS Neglected Tropical Diseases 12: e0006626.

Ndo, C., H. Yamada, D. D. Damiens, S. N'do, G. Seballos, and J. R. L. Gilles. 2014. X-ray sterilisation of the An. arabiensis genetic sexing strain 'ANO IPCL1' at pupal and adult stages. Acta Tropica 131: $124-128$.

Neuenschwander, P., K. Russ, E. Höbaus, and S. Michelakis. 1983. Ecological studies on Rhagoletis cerasi $\mathrm{L}$. in Crete for the use of the incompatible insect technique, pp. 41-51. In R. Calvalloro (ed.), Fruit flies of economic importance. Proceedings of the CEC/IOBC International Symposium. Athens, Greece, 16-19 November 1982. A. A. Balkema, Rotterdam, The Netherlands.

Nikolouli, K., H. Colinet, D. Renault, T. Enriquez, L. Mouton, P. Gibert, F. Sassu, C. Caceres, C. Stauffer, R. Pereira, and K. Bourtzis. 2018. Sterile insect technique and Wolbachia symbiosis as potential tools for the control of the invasive species Drosophila suzukii. Journal of Pest Science 91: 489-503.

O'Connor, L., C. Plichart, A. C. Sang, C. L. Brelsfoard, H. C. Bossin, and S. L. Dobson. 2012. Open release of male mosquitoes infected with a Wolbachia biopesticide: Field performance and infection containment. PLoS Neglected Tropical Diseases 6: e1797.

Oliva, C. F., M. Jacquet, J. R. L. Gilles, G. Lemperiere, P.-O. Maquart, S. Quilici, F. Schooneman, M. J. B. Vreysen, and S. Boyer. 2012. The sterile insect technique for controlling populations of Aedes albopictus (Diptera: Culicidae) on Réunion Island: Mating vigour of sterilized males. PLoS One 7: e49414.

Pal, R. 1966. Genetic control of vectors of disease with special reference to Culex pipiens fatigans. WHO/Vector Control/66.198. World Health Organization, Geneva, Switzerland.

Pal, R. 1974. WHO/ICMR programme of genetic control of mosquitoes in India, pp. 73-95. In R. Pal and M. J. Whitten (eds.), The use of genetics in insect control. Elsevier / North-Holland Publishing Company, Amsterdam, The Netherlands.

Parker, A., and K. Mehta. 2007. Sterile insect technique: A model for dose optimization for improved sterile insect quality. Florida Entomologist 90: 88-95.

Pereira, T. N., M. N. Rocha, P. H. F. Sucupira, F. D. Carvalho, and L. A. Moreira. 2018. Wolbachia significantly impacts the vector competence of Aedes aegypti for Mayaro virus. Scientific Reports 8: 6889.

Portaro, J. K., and A. R. Barr. 1975. "Curing" Wolbachia infections in Culex pipiens. Journal of Medical Entomology 12: 265.

Puggioli, A., M. Calvitti, R. Moretti, and R. Bellini. 2016. wPip Wolbachia contribution to Aedes albopictus SIT performance: Advantages under intensive rearing. Acta Tropica 164: 473-481.

Ranner, H. 1990. Untersuchungen zur Biologie und Bekämpfung der Kirschfruchtfliege, Rhagoletis cerasi L. (Diptera, Trypetidae) - V. Versuche zur Bekämpfung der Kirschfruchtfliege mit Hilfe der Incompatible Insect Technique (IIT). Pflanzenschutzberichte 51: 1-16.

Rasgon, J. L. 2012. Wolbachia induces male-specific mortality in the mosquito Culex pipiens (LIN strain). PLoS One 7: e30381.

Rasgon, J. L., L. M. Styer, and T. W. Scott. 2003. Wolbachia-induced mortality as a mechanism to modulate pathogen transmission by vector arthropods. Journal of Medical Entomology 40: 125-132.

Roubaud, É. 1941. Phénomènes d'amixie dans les intercroisements de Culicides du groupe pipiens. Comptes Rendus Hebdomadaires des Séances de l'Académie des Sciences 212: 257-259.

Ruang-areerate, T., and P. Kittayapong. 2006. Wolbachia transinfection in Aedes aegypti: A potential gene driver of dengue vectors. Proceedings of the National Academy of Sciences of the USA 103: $12534-12539$.

Russ, K., and B. Faber. 1979. The possible use of IIT to control Rhagoletis cerasi L., the European cherry fruit fly in Austria, pages 38-39. In IOBC-WPRS Bulletin, Volume 2, Proceedings of a Joint Meeting of the Working Groups "Biological Control of Olive Pests", "Genetic Control of Rhagoletis cerasi", "Genetic Control of Ceratitis capitata" and "Genetic Methods of Pest Control". 15-20 May 1978. Sassari, Sardinia, Italy.

Scott, M. J., and M. Q. Benedict. 2016. Concept and history of genetic control, pp. 31-54. In Z. N. Adelman (ed.), Genetic control of malaria and dengue. Academic Press, London, UK. 
Segoli, M., A. A. Hoffmann, J. Lloyd, G. J. Omodei, and S. A. Ritchie. 2014. The effect of virusblocking Wolbachia on male competitiveness of the dengue vector mosquito, Aedes aegypti. PLoS Neglected Tropical Diseases 8: e3294.

Shahid, M. A., and C. F. Curtis. 1987. Radiation sterilisation and cytoplasmic incompatibility in a "tropicalized" strain of the Culex pipiens complex (Diptera: Culicidae). Journal of Medical Entomology 24: 273-274.

Sharma, V. P., S. K. Subbarao, T. Adak, and R. K. Razdan. 1979. Integration of gamma irradiation and cytoplasmic incompatibility in Culex pipiens fatigans (Diptera: Culicidae). Journal of Medical Entomology 15: 155-156.

Singh, K. R. P., C. F. Curtis, and B. S. Krishnamurthy. 1976. Partial loss of cytoplasmic incompatibility with age in males of Culex fatigans. Annals of Tropical Medicine and Parasitology 70: 463-466.

Sinkins, S. P. 2004. Wolbachia and cytoplasmic incompatibility in mosquitoes. Insect Biochemistry and Molecular Biology 34: 723-729.

Sinkins, S. P., C. F. Curtis, and S. L. O'Neill. 1997. The potential application of inherited symbiont systems to pest control, pp. 155-175. In S. L. O'Neill, A. A. Hoffmann, and J. H. Werren (eds.), Influential passengers: Inherited microorganisms and arthropod reproduction. Oxford University Press, Oxford, UK.

Subbarao, S. K., C. F. Curtis, K. R. P. Singh, and B. S. Krishnamurthy. 1974. Variation in cytoplasmic crossing type in population of C. p. fatigans Wied. from Delhi area. The Journal of Communicable Diseases 6: 80-82.

Subbarao, S. K., C. F. Curtis, B. S. Krishnamurthy, T. Adak, and R. K. Chandrahas. 1977. Selection for partial compatibility with aged and previously mated males in Culex pipiens fatigans (Diptera: Culicidae). Journal of Medical Entomology 14: 82-85.

Suh, E., D. R. Mercer, Y. Fu, and S. L. Dobson. 2009. Pathogenicity of life-shortening Wolbachia in Aedes albopictus after transfer from Drosophila melanogaster. Applied and Environmental Microbiology 75: 7783-7788.

Teixeira, L., A. Ferreira, and M. Ashburner. 2008. The bacterial symbiont Wolbachia induces resistance to RNA viral infections in Drosophila melanogaster. PLoS Biology 6: e2.

Thomas, V., and K. R. P. Singh. 1977. Comparative susceptibility to Wuchereria bancrofti of Culex pipiens fatigans Delhi strain and of strains cytoplasmically incompatible with it. The Indian Journal of Medical Research 65 (Supplement): 102-106.

Tortosa, P., S. Charlat, P. Labbé, J.-S. Dehecq, H. Barré, and M. Weill. 2010. Wolbachia age-sexspecific density in Aedes albopictus: A host evolutionary response to cytoplasmic incompatibility? PLoS One 5: e9700.

Turley, A. P., M. P. Zalucki, S. L. O'Neill, and E. A. McGraw. 2013. Transinfected Wolbachia have minimal effects on male reproductive success in Aedes aegypti. Parasites \& Vectors 6: 36.

van den Hurk, A. F., S. Hall-Mendelin, A. T. Pyke, F. D. Frentiu, K. McElroy, A. Day, S. Higgs, and S. L. O'Neill. 2012. Impact of Wolbachia on infection with Chikungunya and yellow fever viruses in the mosquito vector Aedes aegypti. PLoS Neglected Tropical Diseases 6: e1892.

Walker, T., P. H. Johnson, L. A. Moreira, I. Iturbe-Ormaetxe, F. D. Frentiu, C. J. McMeniman, Y. S. Leong, Y. Dong, J. Axford, P. Kriesner, A. L. Lloyd, S. A. Ritchie, S. L. O'Neill, and A. A. Hoffmann. 2011. The wMel Wolbachia strain blocks dengue and invades caged Aedes aegypti populations. Nature 476: (7361) 450-453.

Weidhaas, D. E., and J. Seawright. 1976. Comments on the article "Genetic control of mosquitoes" (Laven, 1974), pp. 211-220. In Proceedings Tall Timbers Conference on Ecological Animal Control by Habitat Management, Number 6. Tall Timbers Research Station, Tallahassee, Florida, USA.

Weinert, L. A., E. V. Araujo-Jnr, M. Z. Ahmed, and J. J. Welch. 2015. The incidence of bacterial endosymbionts in terrestrial arthropods. Proceedings of the Royal Society, Series B: Biological Sciences 282: (1807) 20150249.

Werren, J. H., L. Baldo, and M. E. Clark. 2008. Wolbachia: Master manipulators of invertebrate biology. Nature Reviews Microbiology 6: 741-751.

(WHO) World Health Organization. 1964. Genetics of vectors and insecticide resistance. Report of a WHO Scientific Group meeting held in Geneva from 5 to 9 August 1963. Number 268. Geneva, Switzerland. $40 \mathrm{pp}$.

Xi, Z., and S. L. Dobson. 2005. Characterization of Wolbachia transfection efficiency by using microinjection of embryonic cytoplasm and embryo homogenate. Applied and Environmental Microbiology 71: 3199-3204. 
Xi, Z., and D. Joshi. 2016. Genetic control of malaria and dengue using Wolbachia, pp. 305-333. In Z. N. Adelman (ed.), Genetic control of malaria and dengue. Academic Press, London, UK.

Xi, Z., and P. Manrique-Saide. 2018. Scale up from field trial to operation: The combined IIT/SIT approach to eliminate the primary dengue mosquito vectors in China and Mexico. Reunión Nacional del Programa de Enfermedades Transmitidas por Vectores. Los Cabos, Baja California Sur, Mexico.

Xi, Z., J. L. Dean, C. C. H. Khoo, and S. L. Dobson. 2005a. Generation of a novel Wolbachia infection in Aedes albopictus (Asian tiger mosquito) via embryonic microinjection. Insect Biochemistry and Molecular Biology 35: 903-910.

Xi, Z., C. C. H. Khoo, and S. L. Dobson. 2005b. Wolbachia establishment and invasion in an Aedes aegypti laboratory population. Science 310: (5746) 326-328.

Xi, Z., C. C. H. Khoo, and S. L. Dobson. 2006. Interspecific transfer of Wolbachia into the mosquito disease vector Aedes albopictus. Proceedings of the Royal Society, Series B: Biological Sciences 273 (1592): 1317-1322.

Yamada, H., A. G. Parker, C. F. Oliva, F. Balestrino, and J. R. L. Gilles. 2014a. X-ray-induced sterility in Aedes albopictus (Diptera: Culicidae) and male longevity following irradiation. Journal of Medical Entomology 51: 811-816.

Yamada, H., M. J. B. Vreysen, J. R. L. Gilles, G. Munhenga, and D. D. Damiens. 2014b. The effects of genetic manipulation, dieldrin treatment and irradiation on the mating competitiveness of male Anopheles arabiensis in field cages. Malaria Journal 13: 318.

Yamada, H., H. Maiga, J. Juarez, D. De Oliveira Carvalho, W. Mamai, A. Ali, N. S. Bimbile-Somda, A. G. Parker, D. Zhang, and J. Bouyer. 2019. Identification of critical factors that significantly affect the dose-response in mosquitoes irradiated as pupae. Parasites \& Vectors 12: (1) 435.

Yen, J. H., and A. R. Barr. 1971. New hypothesis of the cause of cytoplasmic incompatibility in Culex pipiens L. Nature 232: (5313) 657-658.

Yen, J. H., and A. R. Barr. 1973. The etiological agent of cytoplasmic incompatibility in Culex pipiens. Journal of Invertebrate Pathology 22: 242-250.

Yeung, J. 2018. Australian experiment wipes out over $80 \%$ of disease-carrying mosquitoes. CNN Health. $10^{\text {th }}$ July 2018.

Zabalou, S., M. Riegler, M. Theodorakopoulou, C. Stauffer, C. Savakis, and K. Bourtzis. 2004. Wolbachia-induced cytoplasmic incompatibility as a means for insect pest population control. Proceedings of the National Academy of Sciences of the USA 101: 15042-15045.

Zabalou, S., A. Apostolaki, I. Livadaras, G. Franz, A. S. Robinson, C. Savakis, and K. Bourtzis. 2009. Incompatible insect technique: Incompatible males from a Ceratitis capitata genetic sexing strain. Entomologia Experimentalis et Applicata 132: 232-240.

Zélé, F., A. Nicot, A. Berthomieu, M. Weill, O. Duron, and A. Rivero. 2014. Wolbachia increases susceptibility to Plasmodium infection in a natural system. Proceedings of the Royal Society, Series B: Biological Sciences 281: (1779) 20132837.

Zhang, D., X. Zheng, Z. Xi, K. Bourtzis, and J. R. L. Gilles. 2015a. Combining the sterile insect technique with the incompatible insect technique: I - Impact of Wolbachia infection on the fitness of triple- and double-infected strains of Aedes albopictus. PLoS One 10: e0121126.

Zhang, D., R. S. Lees, Z. Xi, J. R. L. Gilles, and K. Bourtzis. 2015b. Combining the sterile insect technique with Wolbachia-based approaches: II - A safer approach to Aedes albopictus population suppression programmes, designed to minimize the consequences of inadvertent female release. PLoS One 10: e0135194.

Zhang, D., R. S. Lees, Z. Xi, K. Bourtzis, and J. R. L. Gilles. 2016. Combining the sterile insect technique with the incompatible insect technique: III - Robust mating competitiveness of irradiated triple Wolbachia-infected Aedes albopictus males under semi-field conditions. PLoS One 11: e0151864.

Zhang, D., M. Zhang, Y. Wu, J. R. L. Gilles, H. Yamada, Z. Wu, Z. Xi, and X. Zheng. 2017. Establishment of a medium-scale mosquito facility: Optimization of the larval mass-rearing unit for Aedes albopictus (Diptera: Culicidae). Parasites \& Vectors 10: 569.

Zhang, D., Y. Li, Q. Sun, X. Zheng, J. R. L. Gilles, H. Yamada, Z. Wu, Z. Xi, and Y. Wu. 2018. Establishment of a medium-scale mosquito facility: Tests on mass production cages for Aedes albopictus (Diptera: Culicidae). Parasites \& Vectors 11: 189.

Zheng, X., D. Zhang, Y. Li, C. Yang, Y. Wu, X. Liang, Y. Liang, X. Pan, L. Hu, Q. Sun, X. Wang, Y. Wei, J. Zhu, W. Qian, Z. Yan, A. G. Parker, J. R. L. Gilles, K. Bourtzis, et al. and Z. Xi. 2019. Incompatible and sterile insect techniques combined eliminate mosquitoes. Nature 572: 56-61.

Zug, R., and P. Hammerstein. 2012. Still a host of hosts for Wolbachia: Analysis of recent data suggests that $40 \%$ of terrestrial arthropod species are infected. PLoS One 7: e38544. 